\title{
LUMINESCENCE DIGITAL IMAGING MICROSCOPY
}

\author{
Thomas M. Jovin and Donna J. Arndt-Jovin \\ Department of Molecular Biology, Max Planck Institute for \\ Biophysical Chemistry, Postfach 2841, D-3400 Göttingen, \\ Federal Republic of Germany
}

\author{
CONTENTS \\ PERSPECTIVES AND OVERVIEW ........................................................................ 271 \\ PRINCIPLES OF LUMINESCENCE MICROSCOPY .......................................................... 273 \\ Photometric Features of a Luminescence Microscope ......................................... 273 \\ Performance Criteria ..................................................................................... 276 \\ Practical Considerations .................................................................................... 276 \\ SOLID-STATE CHARGE-COUPLED DEVICE CAMERAS FOR LUMINESCENCE IMAGING ............. 276 \\ Properties of CCD Cameras ............................................................................... 277

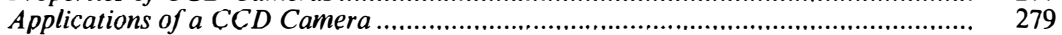 \\ Photobleaching Fluorescence Resonance Energy Transfer Digital Imaging
Microscopy

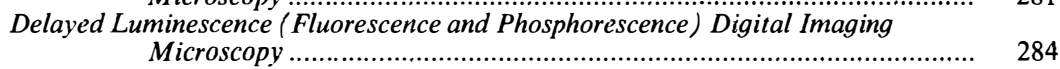 \\ SCANNING LUMINESCENCE MICROSCOPES............................................................ 285

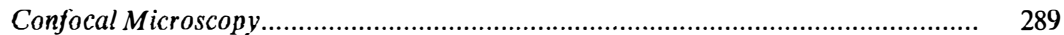 \\ Optical Principles of Confocal Microscopy ..................................................... 289 \\ DATA FLOW IN DIGITAL IMAGING MICROSCOPY …....................................................... 295

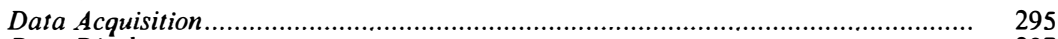

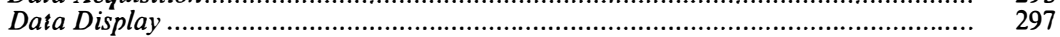

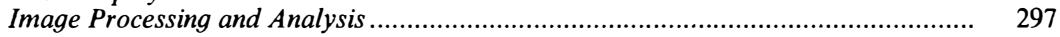

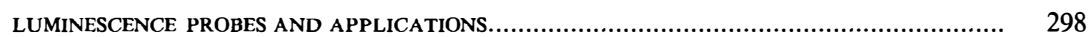 \\ Exploitation of the Photophysics of Luminescence Probes ......................................... 299

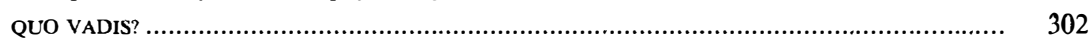

\section{PERSPECTIVES AND OVERVIEW}

The field of optical microscopy is undergoing a technological revolution. The developments underlying this phenomenon are the advent of electrooptical detectors and digitizing electronics, which afford great sensitivity, 
linearity, and dynamic range; the implementation of laser beam and stage scanning systems incorporating confocal optics; the accelerated development of image enhancement, reconstruction, analysis, and display techniques; and the availability of new chemical and immunological probes for specific cellular constituents that can be measured spectroscopically. Of particular benefit to the biologist is the coincidence of these developments with the rapid progress in biotechnology. Thus, molecular genetics can be exploited for genetically modifying macromolecular constituents of the cell or for designing agents that can be introduced externally or internally by physical and biochemical means. In many, if not most, instances the effects of these manipulations have to be assessed in the microscope.

Optical microscopy occupies a unique niche in the array of techniques available for the cell biologist, intermediate between electron microscopy with its high (but myopic) resolution and the methods that examine cell suspensions, e.g. methods based on flow cytometry systems. We have emphasized elsewhere (105) the inherent dichotomy that arises in cell biology with respect to the interactions and functions of individual cells and groups of cells. The available information generally corresponds to the extremes of the resolution scale, i.e. molecules and cells. Numerous approaches exist for studying the essential macromolecules with characteristic dimensions of $<10 \mathrm{~nm}$. However, the development, organization, and functions of complex unicellular and higher organisms involve compartmentalized structures and mechanisms that extend or occur over a micron to centimeter range. The light microscope bridges the enormous gap between molecules and cells, even beyond the nominal spatial resolution limit imposed by the wavelength of light and diffraction phenomena, $\sim 0.3 \mu \mathrm{m}$. Thus, light can be applied not merely as a probe of position and mass, but also as a means of establishing the state of association, the nature of the environment, and the dynamic properties of molecules via a variety of spectroscopic phenomena. These include polarization and other dichroic processes and the transitions between electronic and chemical states $(9,49)$.

We restrict ourselves in this review to microscopy based on the emission of light, classically restricted to fluorescence but more recently expanded to include phosphorescence, bioluminescence (103), and chemiluminescence (119a). In particular, we cover new developments in quantitative measurement using digitized luminescence images. Because of the increasing repertoire of these methods, we favor the general term luminescence digital imaging microscopy (LDIM), rather than the more common designation restricted to fluorescence [FDIM or F-DIM (9)].

Numerous relevant review articles and monographs covering LDIM (1, $9,55,112,181,182,195,196,216,231)$, cell microscopy (109), video- 
enhanced contrast optical microscopy $(4,95,177)$, clinical applications of digital microscopy $(17,94 \mathrm{a}, 100,191)$, and image processing relevant to LDIM $(37,39,57,160,237)$ have appeared recently or are in press. Rosenfeld (159) has compiled a yearly bibliography of new image-processing literature.

\section{PRINCIPLES OF LUMINESCENCE MICROSCOPY}

The classical optical microscope generates images for direct visual observation. The digital microscope based on luminescence phenomena extends the sensitivity and qualitative features of the instrument to the limits imposed by optical and photophysical laws. It is also a quantitative instrument in the photometric sense. The measure of stimulated emission is inherently more sensitive than that of light absorption in terms of the concentration range that can be explored $(91,157)$ and freedom from the effects of inhomogeneous distribution (35). Thus, luminescence shares with several differential quantities (dichroism, birefringence) the enhancement of contrast due to the detection of an absolute rather than a relative signal. In addition, it can provide great specificity and selectivity, e.g. in indirect immunofluorescence, in that the probe and its signal need not be inherent to the property being measured. In a 1985 review Arndt-Jovin et al (9) listed the measurement modalities potentially available for quantitative microscopy based on luminescence. Their implementation has continued unabated in the intervening period.

The practitioner of quantitative microscopy must assess critically $(a)$ saturation effects at high illumination levels (5); (b) irreversible and reversible photobleaching, regarded on the one hand as a useful phenomenon for studying translational and rotational diffusion $(18,49,62,66,107,233)$ and molecular distribution (105; see below) and on the other hand as a nuisance $(20,121,173) ;(c)$ inner filter, scattering, and penetration-dependent effects $(21,170,238)$; $(d)$ fixation versus vital staining strategies; and $(c)$ object motion in relation to the required temporal and spatial resolution.

\section{Photometric Features of a Luminescence Microscope}

The generation of a luminescence image in a microscope comprises three successive processes: (a) illumination (excitation) of an object; (b) emission by the object; and (c) detection of the emission. For biological materials, the second process is stochastic and thus optically incoherent. The illumination may be coherent (i.e. from laser sources), incoherent (as from an arc lamp), or partially coherent, and the polarization state may be random, linear, circular, or elliptical. The excitation process per se is 
intrinsically photoselective in that the probability of exciting a luminophore is given by the projection of the illumination electric vector on the absorption transition moment. The subsequent evolution of luminescence depends upon the spatiotemporal orientation of the corresponding emission transition moment $(49,192)$, the excited-state lifetime(s), and the nature of the photophysical pathway involved, which may involve linear (single-photon) or nonlinear (two-photon or other excited state) processes. As a consequence, to characterize the emission radiance transmitted through the detection path one must specify the local field energy (more precisely the irradiance) and polarization at every position in object space according to the electromagnetic theory appropriate for the illumination system. The nature, localization, and orientation of the luminophore determines the photophysical response. The duration of the excited state relative to the dwell time of observation is of central importance. Thus, the emission of prompt fluorescence is generally characterized by nanosecond lifetimes and is observed in the microscope as a steady-state signal, whereas the fluorescence decay of longer-lived singlets and delayed luminescence from triplet states may be resolved clearly in the time or frequency domains, as shown below. Finally, the optical transfer properties of passive or active polarization-sensitive optical elements must be considered (199). A full discussion of these considerations, crucial as they may be in individual cases, is beyond the scope of this review, but we stress their importance in the quantitative treatment of the recorded data.

To estimate the photometric throughput of a typical luminescence microscope, we regard for the moment a system response that is linear and isotropic, i.e. corresponding to the regime of low, uniform absorption and low irradiance such that saturation (5) is not achieved. (When polarization is an issue, it is assumed that the signals are measured as a function of the global polarization state of the illumination and of the setting of an emission analyzer, so apparent emission anisotropy functions can be calculated.) The excitation energy is absorbed by an individual chromophore in the object field with a probability given by the absorption cross section $\sigma_{\mathrm{A}}\left(\mathrm{cm}^{2}\right.$ molecule $\left.^{-1}\right)$,

$$
\sigma_{\mathrm{A}}=10^{3} \ln (10) \varepsilon_{\lambda} / N \text {, }
$$

where $\varepsilon_{\lambda}$ is the molar absorption coefficient $\left(\mathrm{M}^{-1} \mathrm{~cm}^{-1}\right)$ and $N$ is Avogadro's number. The cross section is a very useful quantity in microscopy, inasmuch as it bypasses the problem of defining a pathlength for absorption. The absorbed energy is converted into emission with a quantum yield $Q_{\text {em }}$, thereby constituting a secondary spherically isotropic point light source with the spectral density of radiant intensity $I_{\lambda}^{\mathrm{em}}\left(\mathrm{W} \mathrm{sr}{ }^{-1} \mathrm{~nm}^{-1}\right.$ molecule ${ }^{-1}$ ). 


$$
I_{\lambda}^{\mathrm{vm}}=E_{\mathrm{cxc}} \sigma_{\mathrm{A}} q(\lambda) Q_{\mathrm{em}}\left(\lambda_{\mathrm{exc}} / \lambda\right) / 4 \pi
$$

where $E_{\text {exc }}$ is the irradiance (W $\mathrm{cm}^{2}$ ) and $q(\lambda)$ is the spectral relative quantum yield distribution function $\left(\mathrm{nm}^{-1}\right)$, a quantity related to the corrected emission spectrum $g(\lambda)$ according to

$$
q(\lambda)=\lambda g(\lambda) / \int g(\lambda) \lambda \mathrm{d} \lambda
$$

In calculating the photon flux at the detector, one cannot assume that the capture of the emitted light into the imaging optics is dictated simply by the numerical solid aperture subtended by the objective, $\pi \mathrm{NA}^{2}$, where NA is the numerical aperture. This formula applies to a Lambertian source (151) but not to an isotropic (diffuse) point radiator. The solid angle of collection for such an object embedded in a medium with refractive index $n$ and located on the optic axis in the focal plane is $2 \pi\left\{1-\left[1-\left(\mathrm{NA} / n_{\max }\right)^{2}\right]^{1 / 2}\right\}$, in which $n_{\max }$ is the highest refractive index in the liquid media, e.g. immersion oil, traversed by the light. The emission is imaged through an optical train with overall transmittance $\tau_{\mathrm{em}}$ and spectral bandwidth $\Delta \lambda_{\mathrm{em}}$ onto the detector, which has a spectral sensitivity $Q_{\lambda}^{\text {det }}$ that determines the relationship between incident photon flux and detected signal (stored charge or current). The energy of a photon at wavelength $\lambda(\mathrm{nm})$ is $\left(2 \times 10^{-16}\right) \lambda^{-1} \mathrm{~J}$. Thus, the photoelectron flux $\Psi_{\mathrm{im}}$ in the image plane (photoelectrons $\mathrm{s}^{-1}$ molecule ${ }^{-1}$ ) is given by

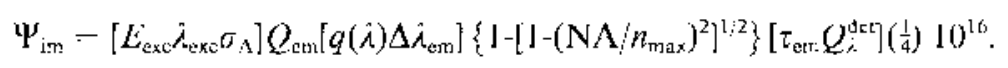

In Equation 4, products within brackets should, if necessary, be evaluated as mean values by integration over the spectral range. Using reasonable values for the various quantities (and fluorescein as a fluorophore), one obtains the useful order of magnitude estimate $\Psi_{\text {im }} \approx 1$ photoelectron $\mathrm{s}^{-1}$ molecule ${ }^{-1}$ per unit irradiance for the conventional fluorescence microscope. With a $63 \times$, NA 1.3 objective and an arc lamp source, we have measured a full-field $(\sim 0.2 \mathrm{~mm})$ irradiance of about $25 \mathrm{~W} \mathrm{~cm} \mathrm{~cm}^{-2}(105)$, corresponding to $\sim 25$ detected photoelectrons $\mathrm{s}^{-1}$ molecule ${ }^{-1}$ according to the above equations. Clearly, higher light levels are required in scanning systems with pixel dwell times of $\sim 0.1-10 \mu \mathrm{s}$. This goal is achieved by using an intense point source image on the object plane, as in the Type Ib (231) conventional microscope, in which the detection of 10 fluorescein molecules $\mu \mathrm{m}^{-2}$ has been reported (152), and in the Type II (231) confocal scanning microscopes discussed below (see Figure 5). 


\section{Performance Criteria}

Owing to the historical emphasis on visual and photographic registration of images, the evaluation of microscope function is often qualitative. However, we can define a list of criteria for the objective specification of microscope performance: (a) acquisition speed and temporal resolution; $(b)$ sensitivity and noise; $(c)$ spectral resolution; $(d)$ spatial resolution; and (e) geometric and photometric linearity, stability, and distortion. Spatial resolution includes optical (diffraction, aberrations), spectroscopic (molecular interactions), and analytical terms. Numerous resolution targets and objects (e.g. beads) as well as image-processing procedures for their assessment are available $(37,65,90,99,101,102,154,227,234,236)$. [The United States National Bureau of Standards will shortly begin to provide standard beads in the $0.1-10 \mu \mathrm{m}$ range for such calibrations (118; R. D. Larrabee, personal communication).] The system performance is best represented by appropriate optical and modulation transfer functions and the intensity point and line spread functions they reflect (see below).

\section{Practical Considerations}

Microscopes used in biology either incorporate the classical configuration or are of the inverted type, with the objectives located below the stage. The latter arrangement facilitates manipulations such as microinjection, additions, and electrode applications, particularly in systems with immobile support stages and mobile optics. However, most laser scanning and confocal systems are of the upright type. The newer generation of planapo objectives, corrected for an infinite conjugate distance, greatly facilitates the introduction of other optical elements in the image-forming optical path (146) by avoiding the limitations imposed by classical tube lengths and convergent beams. In addition, high-NA, low-magnification objectives maximize the efficiency of light collection and image brightness while providing an extended field, a vital feature in applications requiring the examination of large surfaces (153). Still problematical are the limited working distances and the large physical and thermal mass of many objectives, which render difficult the observation of living, thick specimens or of other systems requiring precise thermal control.

\section{SOLID-STATE CHARGE-COUPLED DEVICE CAMERAS FOR LUMINESCENCE IMAGING}

Luminescence is generally a low-light level phenomenon. Thus, its detection in microscopes requires the use of sensitive detectors, of which several are available: $(a)$ video cameras, newly enhanced with high-performance 
gated intensifiers (184); (b) solid-state cameras with charge-coupled devices (CCD) (98) and charge-injection devices (CID) in linear or two-dimensional arrays (24), which provide either video outputs, usually in a TV format, or slow-scanning frame rates along with much greater accuracy and precision, and which can also be intensified (183, 184); (c) microchannel-plate (MCP) cameras with photon-counting position-sensitive detection (PSD) (205) or coupled TV cameras (86); and (d) photodiode arrays with individual signal-processing paths (76).

\section{Properties of CCD Cameras}

Most LDIM systems incorporate TV cameras as detectors. However, such units, e.g. intensified silicon-intensified targets (ISIT), generally have temporal and geometric instabilities, substantial noise, and a nonlinear response to light intensity with a dynamic range limited to 6-7 bits. [Sensitive and accurate digital imaging of fluorescent objects can be obtained in carefully designed systems based on SIT cameras (165).] Because we wish to emphasize the quantitative extraction of luminescence image data we discuss in detail only slow-scan, high-resolution cameras incorporating solid-state CCD arrays; scanning systems with photomultiplier detectors are treated below. The high-performance scientific cameras avoid most of the stated problems. Their rapid development over the last several years has led to markedly improved performance and lower cost, accounting for their increased selection for quantitative LDIM. It is remarkable that the same instruments can be applied to studies of microscopic biological units (cells or particles) and the largest entities in the universe (galaxies).

A CCD sensor consists of a shift register formed by closely spaced metal oxide-silicon (MOS) capacitors that store and transfer electrons introduced optically. The basic advantages of CCD detectors are low background noise in the case of thermoelectrically or cryogenically cooled camera units, lack of geometric distortion, linearity of response over a high dynamic range, and extraordinary sensitivity. Three recent issues of Optical Engineering have covered the state-of-the-art development in this field. Aikens et al (3) have reviewed the specific application of CCD technology to FDIM, and Bilhorn et al $(23,24)$ have compared CCD, CID, and PMT sensors for spectroscopic measurements. Because of the linearity of the CCD response and the very low dark current, noise is dominated by the readout electronics (output amplifier). Thus, the signal can be integrated until the potential well is filled with electrons or as long as required in time-resolved measurements. A recognized problem of vidicon detectors is the phenomenon of "blooming," leakage of overexposed regions into adjacent areas. This difficulty can be circumvented in the CCD array by the inclusion of antiblooming drains between columns 
of pixels, which conduct off excess photogenerated charge from saturated neighboring pixels.

Readout speeds in CCD cameras are determined by the CCD sensor and the associated electronic circuitry, and vary typically from $50 \mathrm{kHz}$ (pixels $\mathrm{s}^{-1}$ ) to $5 \mathrm{MHz}$. The slower rates have precluded the real-time imaging of some processes in living cells. The following technical developments have contributed to speed improvements so that a few systems approach $30-\mathrm{Hz}$ frame rates, albeit with some trade-offs.

BINNING The charges from a number of detector elements in a line are accumulated in a single terminal clcment or "bin," and then transferred in one operation from the bin to the output. Integrating the analog signal on the chip reduces noise more than digital summation, incrcases the uniformity of response, and speeds up the readout of the device. The result is a reduction in spatial resolution unless the image is oversampled. Binning is also useful in the spectral decomposition of emission signals, achieved by optically coupling the camera to the output of a spectrometer. This nonimaging application can be combined with beam scanning instruments such as the laser-based microscopes described below.

SUBFRAME MODE In one operational mode, half of the detector is masked from light and used for the rapid storage of the accumulated contents of the exposed half of the sensor; the readout can then be effected while a second image is collected. Another variation is the use of image-defined subframes for 20-40-ms acquisition (usually dependent upon shutter speed) followed by normal readout of the entire array. This mode is especially useful in LDIM (as shown below for the delayed luminescence measurements) of an object or region that occupies less than $20 \%$ of the entire field.

IMAGE INTENSIFIERS COUPLED TO CCD CAMERAS In one report of an MCP intensifer coupled to a CCD camera through a relay lens (184), geometric distortion was kept to $1.1 \%$ by using only the central portion of the intensifier, gain inhomogeneity was $3 \%$, and gain reproducibility was greater than $99 \%$. With a sacrifice in dynamic range, framing rates of 30 $\mathrm{Hz}$ were achieved. A scientific $\mathrm{CCD}$ camera coupled to a fast-response MCP image intensifier is one of the most sensitive and fast detectors for LDIM. For time-resolved measurements, gating can be achieved down to a few nanoseconds. Ferroelectric liquid crystal electrooptical shutters and polarization controllers are also available.

Some new developments in the CCD field exemplify the extreme design flexibility and future potential of these devices: $(a)$ sensors with a dynamic 
range of $15-16$ bits; $(b)$ improvement in blue and ultraviolet spectral sensitivity by backside thinning and surface treatment or by overlay with a quantum-converting organic phosphor; (c) 64 parallel 128-2K element linear arrays with $20 \mathrm{MHz}$ clock rates and $5 \mathrm{kHz}$ frame rates; (d) CCD photoelements with integrated logarithmic amplifiers offering a 20-bit dynamic range; and $(e)$ sensors with $1.4 \times 10^{6} \sim 7-\mu \mathrm{m}$ pixels.

\section{Applications of a CCD Camera}

The general characteristics of CCD arrays as described above, as well as the ability to integrate for any desired length of time, makes the CCD especially suitable for the high-resolution imaging of multiple low-level fluorescence signals and excited-state processes. In the applications described below, imaging was carried out with a Photometrics (Tucson, Arizona) Series 200 camera system incorporating (a) a mechanical shutter; (b) a thermoelectrically cooled Thomson CSF Model TH7882 CDA CCD sensor with a $576 \times 384$ array of $23-\mu \mathrm{m}$ square pixels, each with a fullwell capacity of $35 \times 10^{4}$ electrons; $(c)$ a dark current of 8 electrons $\mathrm{s}^{-1}$ and a readout noise level of 6 electrons; $(d)$ 14-bit $50-\mathrm{kHz}$ double-correlated analog-to-digital conversion; and $(e)$ a camera controller and image storage and display unit with communication to a central DEC MicroVaxII processing system. (Similar systems are available from other sources.) Our fluorescence microscope (Zeiss Universal) has been further modified to incorporate local or remote computer control of the $x, y, z$ positioning of the stage and of the excitation and emission filters, and mechanical choppers for excitation (laser) and emission.

One example of multiwavelength correlated quantitative LDIM deals with the morphological and temporal features of replication patterns in mouse cells pulsed with 5-bromodeoxyuridine (BrdUrd), a thymidine analog, at different periods of the cell cycle (8; D. J. Arndt-Jovin, T. M. Jovin, and colleagues, manuscript in preparation). Mouse fibroblast cells (line 3T3) were synchronized by serum starvation, released into complete medium, and pulse labeled for 5 min with BrdUrd at various times during S-phase. Cells were stained with a monoclonal antibody to BrdUrd labeled with tetramethylrhodamine isothiocyanate $(\mathrm{Rh})$ and counterstained for DNA with 50- $\mu \mathrm{M}$ mithramycin (MI). The immunofluorescence demarcates the discrete sites of DNA synthesis, and the MI fluorescence shows the quantitative distribution of the DNA within the nucleus. From the dual-wavelength LDIM images for cells in the late S-phase of replication (Figure 1), a clear correlation between condensed heterochromatin and late-S-phase replication patterns is evident.

In the following applications, excited state processes (resonance energy transfer and intersystem crossing) have been exploited in LDIM. 


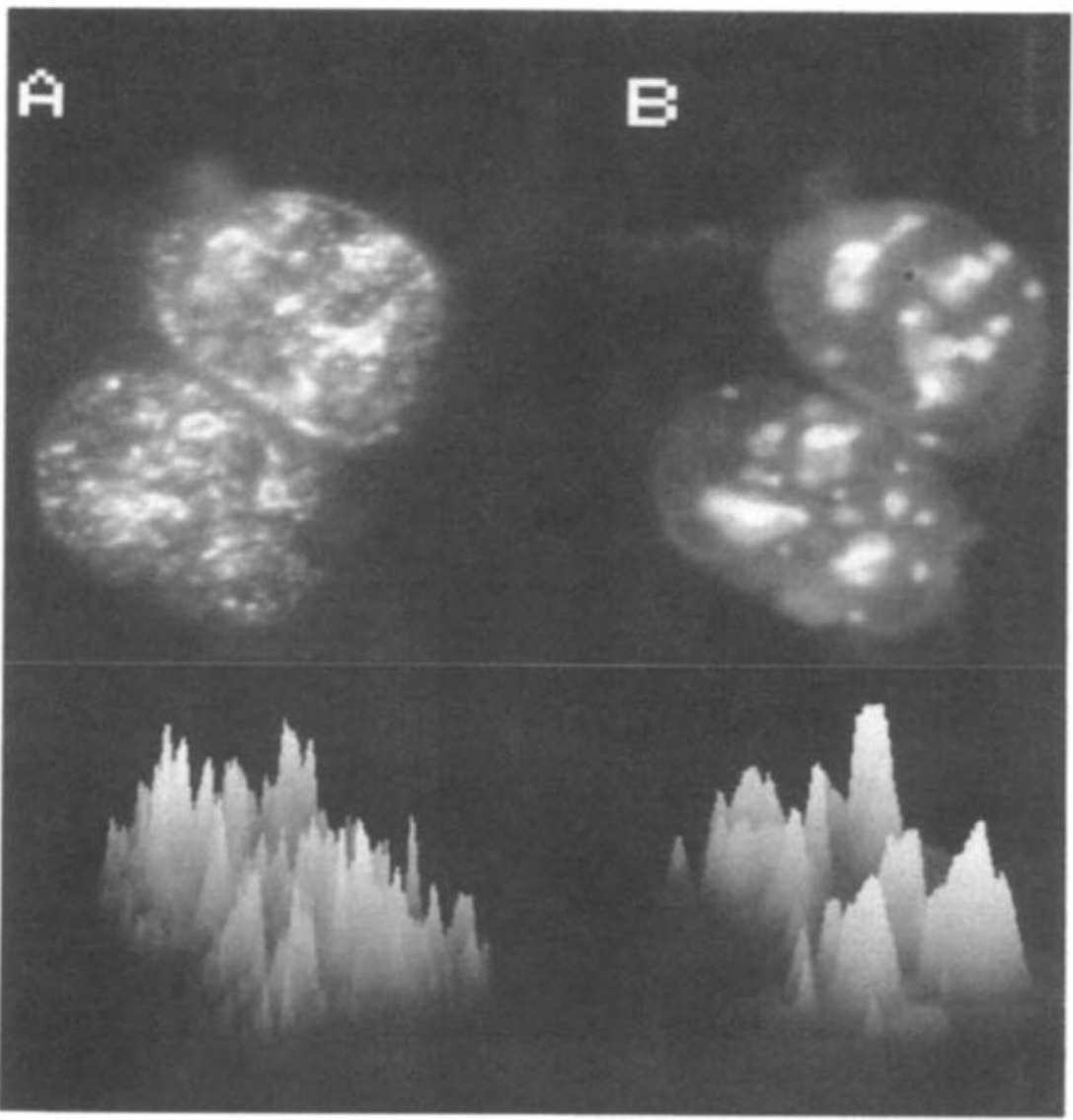

Figure 1 Correlation between replication sites in late S-phase and condensed heterochromatin. LDIM images were acquired using a Zeiss Neofluar $63 \times /$ NA 1.25 oil immersion objective with Optovar set at $1.25 \times$; $50-\mathrm{W} \mathrm{Hg}$ arc lamp excitation source; excitation for $\mathrm{Rh}$ of $546 \mathrm{~nm}$ with emission $>590 \mathrm{~nm}$; excitation for MI at $436 \mathrm{~nm}$ with emission $>450 \mathrm{~nm}$. A Photometrics $\mathrm{CH} 220$ C.CD camera system was used. Images were corrected for bias and excitation field inhomogeneity by division with an image generated from a fluorescent uranium glass. $(A)$ Immunofluorescence (above) and corresponding isometric projection (below) in which height is proportional to grey level. (B) DNA fluorescence of the same cell (above) and corresponding grey-level representation (below). Strong fluorescence intensity of the condensed chromatin correlates with the position of multiple replication complexes appearing as rings. 


\section{Photobleaching Fluorescence Resonance Energy Transfer Digital Imaging Microscopy}

Many mechanisms in cell biology involve the redistribution of macromolecular components and/or the formation of complexes; these include association and processing of molecules involved in signal transduction or ion channels, condensation of DNA and chromatin, modulation of enzymaticactivity by complex formation or structural rearrangement, and fusion and redistribution of membrane components. As stated above, movements on cell surfaces may occur on the scale of microns, but associations of macromolecules take place in the submicron range. How can we probe both of these dimensions simultaneously? One means is measurement of fluorescence resonance energy transfer (FRET) (189) in the light microscope. Energy is transferred from an excited donor fluorophore to an acceptor in close proximity with an efficiency that varies inversely with the sixth power of the separation; the $50 \%$ point (generally $<10 \mathrm{~nm}$ ) depends upon the spectral overlap between donor emission and acceptor absorption and the relative orientation of the chromophores. The consequences of FRET are a reduction of the donor fluorescence lifetime (from $\tau$ to $\tau^{\prime}$ ) and quantum efficiency $Q_{\mathrm{em}}$ (from $Q$ to $Q^{\prime}$ ) such that the transfer efficiency (ET) is given by

$$
\mathrm{ET}=1-\tau^{\prime} / \tau=1-Q^{\prime} / Q .
$$

Until recently, only qualitative FRET microscopy studies had been made $(38,87,88,206,218,219)$, primarily because of difficulties in making the proper corrections for spectral overlap, registration problems with multiple-wavelength measurements, and photobleaching of the donor during the measurement of the sensitized emission.

We have developed a simple method for quantitative FRET measurements in the luminescence microscope that largely avoids the difficulties mentioned above by systematically exploiting the photochemical bleaching of the donor and the ability to integrate an image with precision over several orders of magnitude with the cooled CCD camera (105). This technique, designated photobleaching FRET Digital Imaging Microscopy (pbFRET-DIM), exploits the fact that the time-integrated fluorescence emission obtained upon complete photobleaching of a fluorescent molecule is independent of changes in quantum yield accompanying excited-state reactions such as energy transfer fluorescence resonance, and is independent of the intensity and duration of the excitation (Figure 2). The time constant for photobleaching in the simplest case depends inversely upon the irradiance $E$, the intrinsic photobleaching rate $k_{\mathrm{b}}$, and the lifetime $\tau^{\prime}$ according to 


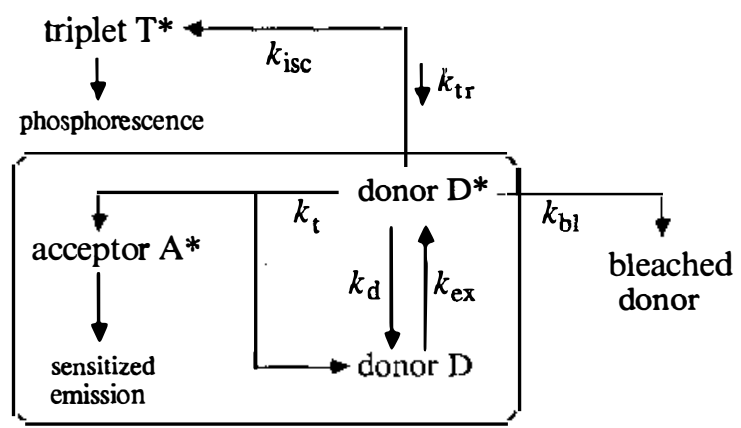

Figure 2 Photophysical scheme for the combined processes of fluorescence energy transfer and donor photobleaching. Rate constants: $k_{\mathrm{ex}}$, donor excitation; $k_{\mathrm{d}}$, decay [including $k_{\mathrm{f}}$ for the radiative (fluorescence) pathway]; $k_{\mathrm{t}}$, nonradiative energy transfer; $k_{\mathrm{b} 1}$, irreversible photobleaching; $k_{1 s c}$, intersystem crossing to the triplet excited state; $k_{\mathrm{tr}}$, thermal reactivation resulting in delayed fluorescence.

$$
\tau_{\mathrm{b} 1} \propto\left[E k_{\mathrm{b} 1} \tau^{\prime}\right]^{-1}
$$

Thus, the time constant for photobleaching increases as the lifetime (and quantum yield) decreases. Accordingly, the integrated fluorescence intensity is invariant and can be used as a measure of the total donor population (105). Since only the donor luminescence is monitored, there is no requirement to register different images, the limitation of the more conventional method based on sensitized acceptor emission (see 105).

The experimental procedure is as follows. From a sample labeled with both acceptor and donor fluorophores, a low-light level image of the donor fluorescence is recorded under nonphotobleaching conditions. The excitation intensity is then increased, and the emission of the donor fluorophore during complete photobleaching is integrated on the CCD sensor (with attenuation of the emission intensity to avoid saturation). A simple ratio of the two images yields the quantum yield of the quenched donor on a pixel-by-pixel basis. The same determination performed on samples devoid of an acceptor, i.e. in the absence of FRET, provides the proportionality constant required to calculate directly from Equation 5 the energy transfer efficiency ET( $i$ ) for each image point $i$ of interest. (Thus pbFRET-DIM constitutes another example of imaging based on quantities derived from dimensionless ratios.) An example of the pbFRET-DIM technique applied to lectin (concanavalin A) receptors on mammalian cells (105) is shown in Figure 3. The high, uniform transfer efficiency reflects the dense surface distributions of glycoproteins and glycolipids in this reference system, which was previously studied extensively by FRET in flow systems and suspension (201). 


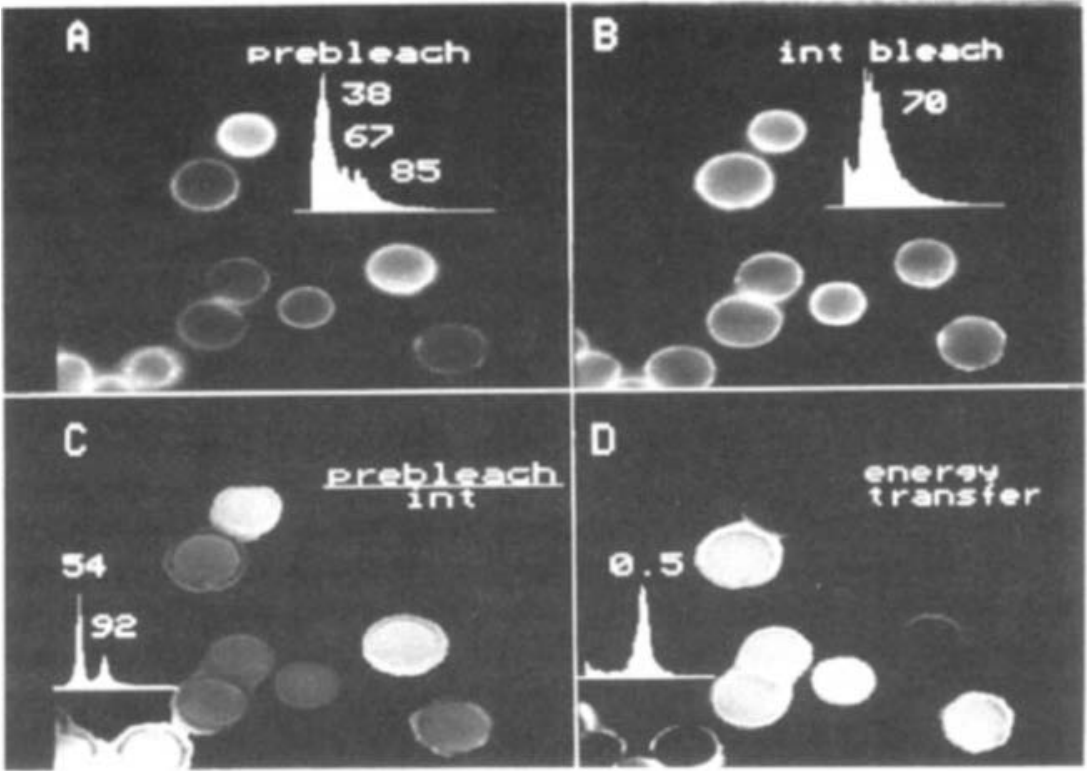

Figure 3 pbFRET images for a mixture of cells labeled with fluorescein-ConA only or with fluorescein-Con $\Lambda$ in combination with tetramethylrhodamine-ConA. Camera and microscope as used in Figure 1, with excitation of fluorescein-ConA at $480 \mathrm{~nm}$ and an emission bandpass of $515-535 \mathrm{~nm}$. $(A)$ The prebleach, $I(d, 0)$, initial intensity image of the donor. A histogram of the grey-level values is shown as an inset. (B) The total integrated donor emission image during bleaching, $I(d$, int $)$, with the corresponding histogram of grey values. (C) $\phi^{\prime}$, the image of the ratio $I(d, 0) / I(d$, int), which is proportional to the quantum yield of the donor. The histogram of the intensities shows two distinct populations with grey values of 54 and 92, corresponding to the doubly and singly labeled cells, respectively. (D) ET(i), the energy transfer efficiency for each image point. The doubly labeled cells show intensity distributions that peak at 0.5 , i.e. $50 \%$ transfer. Data from Reference 105.

The photobleaching rate $\tau_{\mathrm{bl}}(i)^{-1}$ can also be used to derive the energy transfer efficiency. A series of images taken during photobleaching yields the rate constant(s) for photobleaching on a pixel-by-pixel basis, and $\mathrm{ET}(i)=1-\tau_{\mathrm{bl}}(i) / \tau_{\mathrm{b},}^{\prime}(i)$. We are currently applying FRET microscopy in studies of specific cell-surface receptors and antigens. The CCD sensor, because of its stability, linearity, low noise, high dynamic range, and capacity to integrate the optical signals for arbitrary lengths of time, is ideal for these applications.

The above considerations give rise to the concept of a fluorophore as a photophysical catalyst, which converts excitation photons into emission photons for a number of cycles determined by the competing process of irreversible photodecomposition. Thus, one can define the figure of merit 
(fom) for luminescence probes under two circumstances. When the instantaneous emission intensity is given by the product of excitation efficiency and emission efficiency, fom $_{0}=\sigma Q_{\text {em }}$; when total integrated emission is observed, only the photochemical stability is a factor, and fom $\mathrm{f}_{\infty}=k_{\mathrm{f}} / k_{\mathrm{bl}}$. Traditionally, the luminescence microscope is operated in the regime of low excitation intensity so as to minimize photochemical bleaching, and fom $_{0}$ applies. However, if irradiation to extinction with high illumination levels is not contraindicated, the enhanced yield and quantum yield independence reflected in fom $_{\infty}$ (for fluorescein about $10^{4}$ ) implies increased sensitivity and more reliable quantitation. With laser spot illumination, the photobleaching process can be completed in milliseconds, whereas with conventional arc lamps and full-field illumination, seconds to minutes may be required (105).

\section{Delayed Luminescence (Fluorescence and Phosphorescence) Digital Imaging Microscopy}

Many cellular macromolecules are present in low numbers, and their measurement can be obscured by intrinsic fluorescence elicited in living cells as well as by glare and scattering within the microscope. In addition, some biological processes (e.g. signal transduction, growth regulation) involve molecular motions such as rotational diffusion, which for membrane-associated components occur in the millisecond-microsecond domain (107) and cannot be monitored with the decay of the excited singlet state in the nanosecond domain. For cell suspensions, such measurements have been made by the polarized delayed luminescence (phosphorescence or delayed fluorescence) of probes with long-lived triplet states potentiated by heavy-atom substituents (107; Figure 2). (The triplet state is quenched by oxygen; thus measures for its exclusion are usually required.) Such determinations have not yet been carried out in the microscope, except by using the complementary phenomenon of ground-state depletion monitored by prompt fluorescence $(66,233)$. Although the observation of longlived phosphorescence of biological materials in the microscope was reported as early as $1942(83)$, the phenomenon had not until recently been investigated with modem digital imaging systems.

The first images obtained through delayed luminescence digital imaging microscopy (deLDIM) were recently recorded using an acousto-optic modulator or mechanical chopper to modulate a laser or lamp source, and a second phase-locked chopper in front of the CCD camera described above (105a; G. Marriott, R. M. Clegg, D. J. Arndt-Jovin \& T. M. Jovin, in preparation). The emission can be integrated in a selected time window of phase and width adjusted to correspond either to prompt fluorescence or to the delayed luminescence, whose lifetime(s) can be evaluated on a 
pixel-by-pixel basis. In one application, the metachromatic dye acridine orange displayed a long $(1 \mathrm{~ms})$ delayed fluorescence and phosphorescence lifetime when bound to cellular macromolecules such as DNA and RNA and in the absence of oxygen. The delayed luminescence images of both polytene and diploid chromosomes stained with acridine orange were obtained, with signals relative to the corresponding fluorescence image (at zero phase shift) of $\sim 0.1 \%$.

Another significant initial application of deLDIM has been the imaging of sites undergoing DNA replication in living cells. The thymidine analog BrdUrd was again used. Binding of acridine orange to sites of $\mathrm{Br}$ incorporation in DNA leads to a pronounced enhancement of the yield and prolongation of the lifetime of delayed luminescence, as determined in previous solution studies (42). In the microscope, the delayed fluorescence signal at sites of replication was $5 \%$ that of the corresponding prompt fluorescence, thus providing high contrast and selectivity (105a; Figure 4). The immunofluorescence procedure described previously (Figure 1) is also highly specific, but it is applicable only to fixed cells. Another fluorescence dye, the DNA minor-groove ligand bisbenzimidazole BBI-342, stains living cells (7) but is relatively quenched at sites of $\mathrm{Br}$ incorporation, which thus cannot be perceived in the microscope image because of low contrast.

The pbFRET-DIM and deLDIM techniques have obvious potential for extension to other situations of interest, e.g. those involving DNA hybridization or virus detection. Appropriate probes, including those based on the long-lived fluorescence emission of lanthanides, are currently under development. The temporal resolution of deLDIM can be extended to the submicrosecond range by the use of gated intensifiers or incorporated into the beam scanning microscopes described in the next section.

\section{SCANNING LUMINESCENCE MICROSCOPES}

In the conventional fluorescence microscope the entire field is illuminated and the total corresponding emission is either observed visually, photographed, or imaged onto a two-dimensional detector (camera). The field of observation of highly automated, multiparameter systems (153) is selected by computer-controlled stage movement, which permits a scan of the entire object, e.g. a slide. In the following discussion, however, we restrict the definition of scanning microscopes to those instruments that operate on the principle of image formation by illumination and detection on a point-by-point $(11,134,231)$ or line-by-line basis $(236)$. Such systems are of three types. 


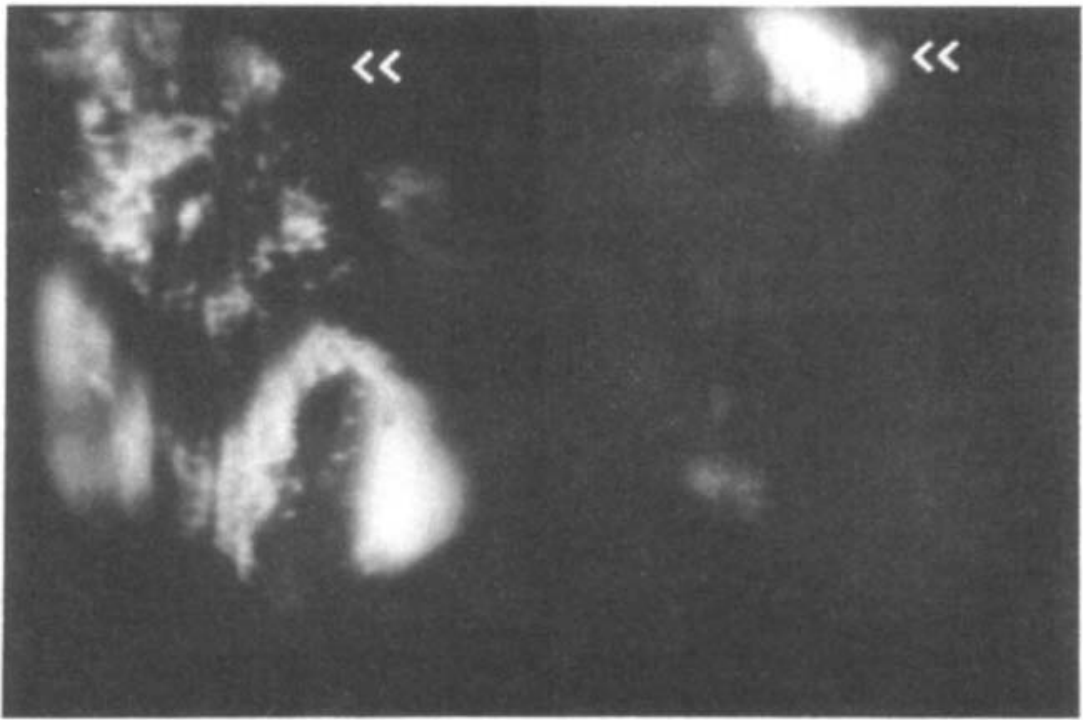

Figure 4 Prompt and delayed luminescence digital images of living cells stained with acridine orange. Nonsynchronized cells were grown in BrdUrd for $2 \mathrm{hr}$ and then chased in normal medium for $4 \mathrm{hr}$. Cells were stained with $50 \mu \mathrm{M}$ acridine orange, washed, suspended in saline buffer containing an enzymatic oxygen scavenger, and measured. Camera and microscope were as in Figure 1, with excitation at $436 \mathrm{~nm}$ and emission $>515 \mathrm{~nm}$, but with a mechanical chopper in the excitation path and a second chopper with tunable phase in the emission path. Chopping frequency $527 \mathrm{~Hz}$, pulse width $200 \mu \mathrm{s}$. Left: Prompt fluorescence image with free-running emission chopper camera integration for $1 \mathrm{~s}$. Strong cytoplasmic fluorescence of the lysozomal particles is scen in five cells. Right: Delayed luminescence image of the same field with the emission chopper phase locked to $40^{\circ}$, integration $40 \mathrm{~s}$. A strong phosphorescence signal is seen in one cell nucleusundergoing mitosis ( $\ll)$, indicating that BrdUrd was incorporated into the DNA during the pulse labeling. The delayed luminescencc signal in the lysozomal particles is $<0.05$ that of the mitotic nucleus, and no other nuclei are visible.

1. In stage (or object) scanning systems, the object is moved relative to a stationary, on-axis, focused beam. ${ }^{\prime}$ Stage scanning instruments require only good paraxial performance; therefore the flat-field requirement is relaxed. Thus, one can optimize objectives for high NA and long working distance, which are important in the examination of thick specimens. The slowness of object scanning can be overcome with a stage based at least partially on piezoelectric elements (137; see Table 1).

\footnotetext{
'This classification could be extended to include flow cytometers and sorters based on hydrodynamic focusing of suspended cells and particles transported through a focused laser beam (211). Although these flow systems have zero or limited [as in slit-scanning (47)] spatial resolution because of their detection mechanisms, they can be incorporated into existing microscopes $(71,185,211)$ and have been operated in a confocal mode with multiple laser sources and detectors $(186,211)$.
} 
2. In beam scanning systems, the focused illumination (excitation) beam, usually from a laser, is moved relative to the object by means of tandem galvanometric mirrors or by a combination of mirrors with acousto-optical deflectors or rotating polygons. One fast dimension $(x)$ corresponds to individual scan lines, and a second, slower dimension $(y)$ defines the frame format. In addition, some of these systems offer $z$-axis (axial) scanning through the action of stepping motors or piezoelectric devices attached to either the stage or the objective. An alternative, unique design is a direct-viewing confocal microscope based on tandem mechanical flying-spot scanning with incoherent light.

3. In detector scanning systems, a linear array detector is either transported physically in the orthogonal direction or coupled to a rotating mirror or scanned stage for the generation of the second dimension (19, 236).

Representative microscopes used for the measurement of fluorescence according to the first two of the above techniques for optical scanning are summarized in Table 1. These systems image luminescent objects confocally, i.e. with high resolution, and also incorporate other (not necessarily confocal) contrast modes based on reflection and transmission. Differential interference contrast (DIC) is particularly effective in combination with fluorescence. The initial pioneering efforts in numerous research laboratories have stimulated the development of mature yet continuously evolving commercial instruments. In addition, other laser scanning microscopes have been reported for measurements of transmitted and reflected light with sensitivity for differential amplitude (169), birefringence (82), optical amplitude and phase (108), acoustic amplitude and phase (161), and phase and polarization in a differential mode (142) or with high contrast from optical heterodyning (166). Real-time response has been achieved in some systems (190; see also Table 1).

Optical techniques combined with other scanning and imaging modalities can offer superresolution (in terms of lateral resolution and depth of field) beyond the limit imposed by the diffraction of light. Laser scanning versions of the following instruments either exist or should be feasible. (a) The video-enhanced contrast optical microscope achieves resolution beyond the Rayleigh limit by electronic background compensation and optical phase shifting $(4,95)$. (b) In the laser electron microscope (LEM), an electron scanning beam elicits a cathodoluminescence signal from a sample overlayed with a phosphor and illuminated with an infrared laser; the resolution is $\sim \lambda / 10(131)$. (c) The near-field scanning optical microscope (NSOM) achieves high spatial resolution by imaging with the near field of a submicron physical aperture; fluorescence detection is being 
Table 1 Scanning microscopes for fluorescence (luminescence) measurements

\begin{tabular}{|c|c|c|c|c|c|c|c|c|}
\hline \multirow[t]{2}{*}{$\therefore=.=$} & \multicolumn{2}{|c|}{$\overline{\overline{ }}=\overline{=}=$} & $-\overline{-}-$ & $=$ & 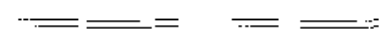 & \multicolumn{2}{|l|}{$\therefore$} & \multirow[t]{2}{*}{$=\overline{=}$} \\
\hline & & & Scan & Scanni & g elements $s^{c}$ & & & \\
\hline System ${ }^{a}$ & Light source & UV & $x, y$ & $x, y$ & $z$ & $\begin{array}{c}\text { - Confocal } \\
\text { optics }\end{array}$ & Detector $^{d}$ & References \\
\hline$-\square-\bar{C}$ & $\cdots--$ & - - & - & - & -“. & --. & $-\overline{\mathrm{DN}}$ & 10 \\
\hline Multipoint FRAP & Laser & - & B & Galv $(x)$ & - & + & PM & 113 \\
\hline $\operatorname{ACAS}(M)$ & Laser & - & S & $\mathrm{Sm}$ & - & & PM & 167 \\
\hline CSLM & Laser & + & $S$ & EMVS & Stage (PZT) & + & PM & 208,210 \\
\hline SOFM & Laser & & S & EMVS & $-\cdots-$ & + & PM & 44 \\
\hline EMBL CSSLM & Laser & + & $S$ & Galvs & Stage (PZT) & + & PM & 188,225 \\
\hline EMBL CBSLM & Laser & - & B & Galv & Stage (PZT) & + & PM & 187 \\
\hline $\operatorname{CBLSM}(H)$ & Lasers (2) & - & B & Galv(s) & Stage (PZT, M) & + & PM & 224 \\
\hline $\operatorname{TCSM}(\mathrm{TRLSM})(T)$ & Lamp & + & B & Perforated disk & Objective (PZT) & + & Cam & 27,149 \\
\hline $\operatorname{CLSM}(Z)$ & Lasers (2 or 3 ) & + & B & Galv & Stage (SM) & + & $\mathrm{PM}$ & $106,152,158,226$ \\
\hline PHOIBOS $(S)$ & Laser & - & S & Mirrors & Stage (SM) & + & PM & 13 \\
\hline MRC-Lasersharp (B) & Laser & - & $\mathrm{B}$ & Polygon/Galvs & Stage (M) & + & PM & 223 \\
\hline Ultrafast LSM & Lasers (2) & + & $\mathrm{B} / \mathrm{S}$ & Polygon/Galv & Objective (LA) & + & PM & 172 \\
\hline LSM & Laser & -. & $\mathrm{B}$ & Galvs & Stage & + & PM & 193 \\
\hline TNO-CLSM & Laser & & B & $\mathrm{AOD} / \mathrm{Galv}$ & Stage (PZT) & + & PM & 58 \\
\hline
\end{tabular}

${ }^{a}$ Original designation or standard (188) acronym: C, confocal; CB, confocal beam; CS, confocal stage; SLM, beam scanning; LSM, stage scanning. Firms associated with the commercialization of the instruments are indicated by letters in parentheses: Meridian $(M)$, Heidelberg Instruments $(H)$, Tracor Northern $(T)$, Carl Zeiss $(Z)$, Sarastro $(S)$, Biorad $(B)$

${ }^{\mathrm{b}} \mathrm{B}$, beam scanning; S, stage scanning. Frame formats are variable, typically $512 \times 512$. Representative single frame times are in the range $0.1-2 \mathrm{~s}$, but some of the instruments (TCSN, Ultrafast LSM, TNO-CLSM) offer video-rate acquisition. Those with PZT- $z$ drives can perform $x, z$ as well as $x, y$ and $x$ scanning.

${ }^{\mathrm{c}}$ Scanning in the focal plane (line axis, $x$; frame axis, $y$ ) and along the optic axis $(z)$. Galv(s), galvanometer(s); SM, stepping motor; EMVS, electromechanical vibrators; AOD, acousto-optic deflector; PZT, piezoelectric translator; M, motor; LA, linear actuator.

'PM, photomultiplier; Cam, two-dimensional video or slow-scan camera. 
attempted with this instrument (96). It can be anticipated that optical detection will be feasible with additional members of the rapidly proliferating family of scanning tip microscopies (197). (d) In total internal reflection fluorescence (TIRF) microscopy $(14,69)$, focal cellular contacts with a substrate or, in another intriguing application, individual motile virus particles (92) are visualized by luminescence excited in the submicron evanescent field. $(e)$ Photoelectron microscopes image photoelectrons of objects irradiated with ultraviolet light; the current resolution is $\sim 10 \mathrm{~nm}$ (75). Finally, because of the similarities in the processes of luminescence and elastic scattering, we mention the Raman scattering microscope, which can be operated in both resonance (16) and coherent anti-Stokes (59) modes. Confocal configurations should be possible.

\section{Confocal Microscopy}

The contemporary confocal optical microscope represents the implementation of concepts related to superresolution that span more than two decades $(45,73,125,126,139,231)$. The experience gained with the early confocal scanning microscopes $(29,60,175)$ and fluorescence microscopes based on laser excitation $(22,62,110,114)$ led to the evolution of the first generation of confocal fluorescence laser scanning systems $(44,210)$. The virtues of laser scanning systems in general are point illumination with minimization of stray light, glare, interf erence phenomena, and incidental photobleaching; flexible selection of the photophysical process leading to image formation with control of spectral range, modulation, and polarization; electronic control of magnification (zooming) without loss of contrast; electronic control of detector bandwidth, background, and gain; high spatial resolution; and preservation of geometric registration in multiparameter imaging. The particular advantages of confocal configurations are described below.

\section{Optical Principles of Confocal Microscopy}

All of the characteristic features of the confocal microscope can be derived (231) from the principles of Fourier optics (26, 37, 73; References 140 and 227 are particularly useful guides for optical applications). The distinctive components are a point source for illumination and a point detector backimaged on the same volume element (Figure 5). The most commonly used epi-illumination configuration is a shared-aperture system in which the same objective is used for excitation and collection of the luminescence. Instruments based on stage (object) scanning are axially centered and thus exhibit optimal paraxial performance. Beam scanning systems incorporate a descanning mechanism (Table 1) such that all object points are imaged on a stationary, axially central field stop defining the active detector 


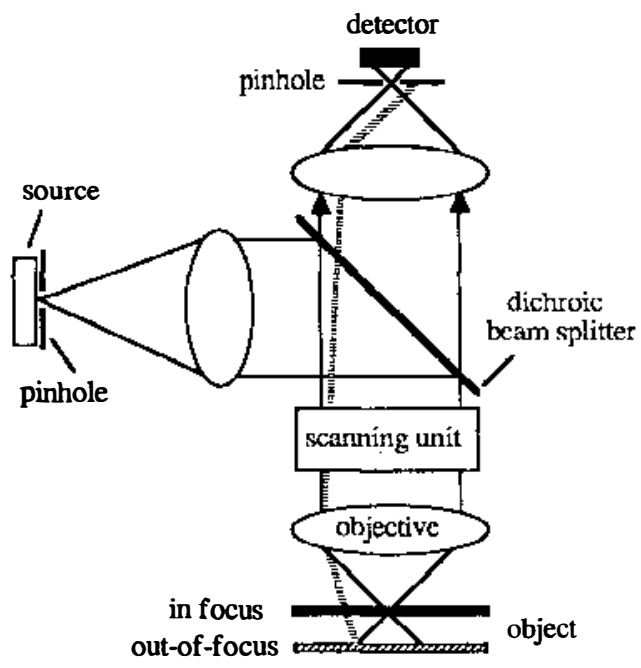

Figure 5 Schematic diagram of a confocal laser scanning microscope. The source can be a lamp or a laser. The emission from the out-of-focus object planes (striped path) is blocked by the detector pinhole, which is lacking in the conventional scanning microscope.

surface, taken here to be circular with radius $r_{\mathrm{d}}$. The ideal point source uniformly fills the entrance pupil of the aberration-free illumination objective of numerical aperture $\mathrm{NA}_{\text {exc }}$. We assume an objective with a telecentric aperture stop, insuring that the scanning pencil is normal to the object field with no vignetting.

For moderate NA values, e.g. $<0.6$, the distribution of radiant energy near the focus point is given by the far-field scalar Fraunhofer diffraction theory in terms of the normalized dimensionless optical coordinates $v$ and $u$, normal to and along the local optic axis, respectively (i.e. corresponding to the momentary scan position):

$$
\begin{aligned}
& v=r\left(2 \pi / \lambda_{\text {exc }}\right) \mathrm{NA}_{\text {exc }}, \\
& u=z\left(2 \pi / \lambda_{\text {exc }}\right) \mathrm{NA}_{\text {exc }}^{2} / n_{\text {max }} .
\end{aligned}
$$

Here $r$ and $z$ are the radial distance from the optic axis and the displacement from the focal plane (to either side), respectively; $\lambda_{\text {exc }}$ is the illumination wavelength in air; and $n_{\max }$ is the operative refractive index defined earlier. According to assumptions made above, the system is considered linear with respect to the irradiance and the luminescence response thereto, and it is also considered shift invariant $(37,73)$. Young $(235)$ has given a 
particularly cogent account of the fluorescence microscope in terms of linear system theory. The incoherence of the photophysical emission process enhances resolution because intensities and not amplitudes add in the image $(139,231)$. Thus the distribution of elicited luminescence radiance is proportional to the intensity point spread function, equal to the modulus squared of the amplitude point spread function $\mathbf{h}(u, v)$, the two-dimensional Fourier transform of the pupil function. If the pupil function is uniform, radially symmetrical, and circular, $\mathbf{h}^{2}$ is given in the focal plane $(u, z=0)$ in normalized form by the familiar Airy function,

$$
\mathbf{h}^{2}(0, v)=\left[2 J_{1}(v) / v\right]^{2}
$$

where $J_{1}(v)$, the Bessel function of the first kind and order one, consists of concentric rings with a central maximum and the first zero located at $v=1.22 \pi$. This value corresponds to the Rayleigh definition of lateral resolution $\left(\Delta=0.61 \lambda_{\text {exc }} / \mathrm{NA}_{\text {exc }}\right.$; Equation 7$)$ for the Type Ib scanning luminescence microscope described earlier, i.e. one with an unrestricted detector $\left(r_{\mathrm{d}} \rightarrow \infty\right)$ and a response limited by the entrance aperture. Along the optic axis, $\mathbf{h}^{2}(u, 0)$ has the normalized form

$$
\mathbf{h}^{2}(u, 0)=\operatorname{sinc}^{2}(u / 4 \pi) \text {, }
$$

also a damped oscillatory function with a constant period of $4 \pi$; this value constitutes a measure of axial resolution according to the Rayleigh criterium. Applying these (arbitrary) definitions, the ratio of lateral to axial resolution is given by $\Delta v / \Delta u=0.3$; alternatively, in terms of real coordinates in the focal plane, $\Delta r / \Delta z=0.3 \mathrm{NA}_{\text {exc }} / \boldsymbol{n}$. (Thus the ratio is only 0.13 for a NA of 0.6 in water!) The greatly reduced axial resolution (poor depth discrimination) and resultant out-of-focus contributions constitute major problems in conventional microscopy.

For an extended object described by $\mathbf{t}(z ; x, y)$, in which the $x, y$ Cartesian coordinates define the radial position $r$, the spatial distribution of elicited luminescence is given by the convolution (denoted by the operator *) $\mathbf{h}^{2}\left(u_{\mathrm{exc}}, v_{\mathrm{exc}}\right) * \mathbf{t}(z ; x, y)$. The detection process can now be considered in analogous fashion in terms of a response function $\mathbf{h}^{2}\left(u_{\mathrm{en}}, v_{\mathrm{en}}\right)$ for the collection objective with a numerical aperture $\mathrm{NA}_{\mathrm{em}}$ determined by some aperture stop. (Thus, even in an epi-illumination system with a single objective, $\mathrm{NA}_{\mathrm{exc}}$ and $\mathrm{NA}_{\mathrm{em}}$ can differ.) We also define a field-stop function $\mathrm{d}\left(u_{\mathrm{em}}, v_{\mathrm{em}} ; r_{\mathrm{d}} / M\right)$, in which $M$ is the objective magnification, such that the overall optical response function corresponding to the image signal is given by the product of two convolutions $(227,231)$ :

$$
I_{\mathrm{im}}(z ; x, y) \propto \phi\left\{\mathbf{h}^{2}\left(u_{\mathrm{exc}}, v_{\mathrm{exc}}\right) * \mathbf{t}(z ; x, y)\right\}\left\{\mathbf{h}^{2}\left(u_{\mathrm{em}}, v_{\mathrm{em}}\right) * \mathbf{d}\left(u_{\mathrm{em}}, v_{\mathrm{em}} ; r_{\mathrm{d}} / M\right)\right\} .
$$


Here $\phi$ is a composite constant containing the product of all the linear transfer factors and irradiance described earlier (Equation 4), and the normalized radial coordinates are defined in terms of the numerical apertures and wavelengths for excitation and emission (Equations 7 and 8; in general, $\lambda_{\mathrm{em}}>\lambda_{\text {exc }}$ ). Note that Equation 11 assumes that the illumination and collection objectives are truly confocal in the sense of having coincident optic axes and focal planes. The back-projection onto the focal plane of a circular aperture with radius $r_{d}$ corresponding to the active surface of the detector is the circle function $\operatorname{circ}\left[r /\left(r_{\mathrm{d}} / M\right)\right]$. This function can be used to calculate analytically the explicit dependence of the focused image intensity on detector size (229). Equations 12 and 13 are the more general solutions for the image intensity point spread function for the conventional Type $\mathrm{Ib}$ scanning microscope, $I_{\mathrm{im}}^{\mathrm{i}}$, and for the Type II (confocal) scanning microscope, $I_{\mathrm{im}}^{\mathrm{cf}}$.

$$
\begin{aligned}
& I_{\mathrm{im}}^{\mathrm{c}}(z, r)=\lim _{r_{\mathrm{t} t \rightarrow \infty} \rightarrow \infty} I_{\mathrm{im}} \propto \phi \mathbf{h}^{2}\left(u_{\mathrm{exc}}, v_{\mathrm{exc}}\right)
\end{aligned}
$$

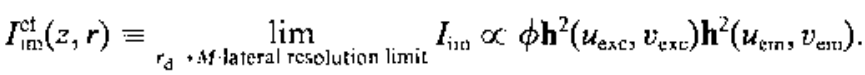

The latter solution is achieved at the lower limit for $r_{d}$ imposed by the lateral resolution of the optical system.

All of the unique virtues of the confocal scanning systems are a consequence of Equation 13, in which the operative point spread function is simply the product of the two individual response functions for illumination and collection. As a consequence, $I_{\text {im }}^{\text {cf }}$ decays more rapidly than $I_{\mathrm{im}}^{\mathrm{c}}$ in the lateral and axial directions, which leads to the following advantages $(46,208,230,231)$ :

1. Increased lateral resolution, up to a factor of 1.3 using the two-point Rayleigh criterion applied to the function $\mathbf{h}^{4}$ (Equation 9) for the limit case of $\lambda_{\mathrm{em}}=\lambda_{\text {exc }}$ and $\mathbf{h}^{2}\left(0, v_{\mathrm{exc}}\right)=\mathbf{h}^{2}\left(0, v_{\mathrm{em}}\right)$.

2. Increased axial resolution, as seen from the fact that products of $\operatorname{sinc}^{2}(x)$ functions (Equation 10) decay more rapidly than $\operatorname{sinc}^{2}(x)$, even though the zero crossings are unchanged.

3. Apodization, i.e. attenuation of the side lobes in the focal plane intensity distribution.

4. Suppression of glare and scattering because of the restricted detector area. The contrast enhancement gained by rejection of parasitic signals (151) comes at the price of increased shot noise to the degree that the desired signals are also attenuated $(178,230)$.

5. Suppression of out-of-focus contributions.

Feature 5 is of paramount importance in the confocalmicroscope in that it permits the facile examination of thick specimens, if they are sufficiently 
transparent. From Parseval's theorem (conservation of radiant flux), the integrated emission corresponding to a luminescence point is invariant upon displacement to either side of the focal plane $(z, u \neq 0)$ if it is collected by a large area detector as in the Type Ib microscope (Equation 12). The defocused image appears as a uniform background with loss of contrast. While compensation is feasible, image deconvolution as an initial step in the three-dimensional reconstruction from optical slices constitutes a major problem, as discussed further below. The confocal microscope exhibits a very different behavior, in that the integral of $I_{\mathrm{im}}^{\mathrm{cf}}(z, r)$ over $r$ diminishes rapidly on either side of the focal plane $(43,155,178,229-231)$, reflecting the physical interception of the out-of-focus components by the detection pinhole (Figure 5). This pronounced depth discrimination is a phenomenon distinct from the moderate decrease in depth of field [in itself an ambiguous quantity $(150,151,174,178)]$, discussed under point 2 above. For example, depth discrimination is enhanced prior to the increase in resolution as the detector area is decreased $(178,229,230)$; this property accounts for the good performance of the tandem scanning microscope despite the relatively large pinhole sizes $(\sim 60 \mu \mathrm{m})$ currently used in that instrument (27).

The normalized Fourier transf orm of the intensity point spread function (or of the first derivative of the intensity line spread function corresponding to a measured edge function) is the optical transfer function (OTF). In the frequency domain, the OTF acts as a filter operating on the transform of $\mathbf{t}(z ; x, y)$, the distribution of luminophores in the object. The modulus of the OTF, the modulation transfer function (MTF), is the most convenient measure of the spectral bandwidth characteristic of the system. Its most important characteristics are unit value at zero frequencies and an absolute passband limitation, corresponding to the finite extent of the entrance and exit pupils of the system. The lateral cutoff frequency for the nonconfocal case is given by $f_{\max }=2\left(\mathbf{N A}_{\text {exc }} / \lambda_{\text {exc }}\right)$, and that for the confocal case by $f_{\max }=2\left(\mathrm{NA}_{\mathrm{exc}} / \lambda_{\mathrm{exc}}+\mathrm{NA} \mathrm{A}_{\mathrm{em}} / \lambda_{\mathrm{em}}\right)$. According to this resolution criterion, a maximal increase in spatial bandwidth of up to two fold can be achieved $\left(\right.$ for $\left.\mathrm{NA}_{\mathrm{exc}}=\mathrm{NA}_{\mathrm{em}}\right)$.

We briefly mention some other characteristics of confocal scanning systems. (a) Changing objectives (NA, magnification) and detector pinhole size affects the degree of confocality. (b) Depending upon its spatial distribution, a primary luminescent source, e.g. one based on bioluminescence $(67,84,103)$ or chemiluminescence $(119 a)$, may yield images that have higher resolution than those obtained by conventional fluorescence in the microscope with a point detector. (c) Apodization by illumination with a full-field Gaussian laser beam reduces spatial resolution; $50 \%$ truncation is almost equivalent to a homogeneous distribution (115). (d) To avoid 
degradation of performance due to spherical aberration of high-NA objectives, careful adjustment of the immersion fluid refractive index may be required (90). (e) Operation at high NA leads to field distributions around the focal plane that deviate substantially from predictions from scalar Fraunhofer diffraction theory $(63,122,130,176,200)$. The point of maximal intensity is shifted toward the entrance pupil (focal shift), the symmetry about the focal plane is decreased, and polarized field components arise along all axes. These effects may influence quantitative interpretations and merit further investigation. The display and processing of images obtained by optical sectioning with the confocal microscope are considered below.

With the availability of commercial instruments, data are emerging on the interactions of macromolecular structures in tissues and organisms, systems heretof ore only accessible by thin-section electron microscopy. A number of recent symposia have considered applications of confocal laser scanning microscopy (CLSM) to structural problems in cell biology (15, 182).

An example from our laboratory is given in Figure 6. The distribution of late S-phase replication sites in a mouse fibroblast cell nucleus was visualized with fluorescein-labeled antibody against the thymidine analog BrdUrd in the same cells described in Figure 1. With a modified Zeiss CLSM $(106,158), 6-8$ optical sections were acquired at $0.4 \mu \mathrm{m}$ intervals

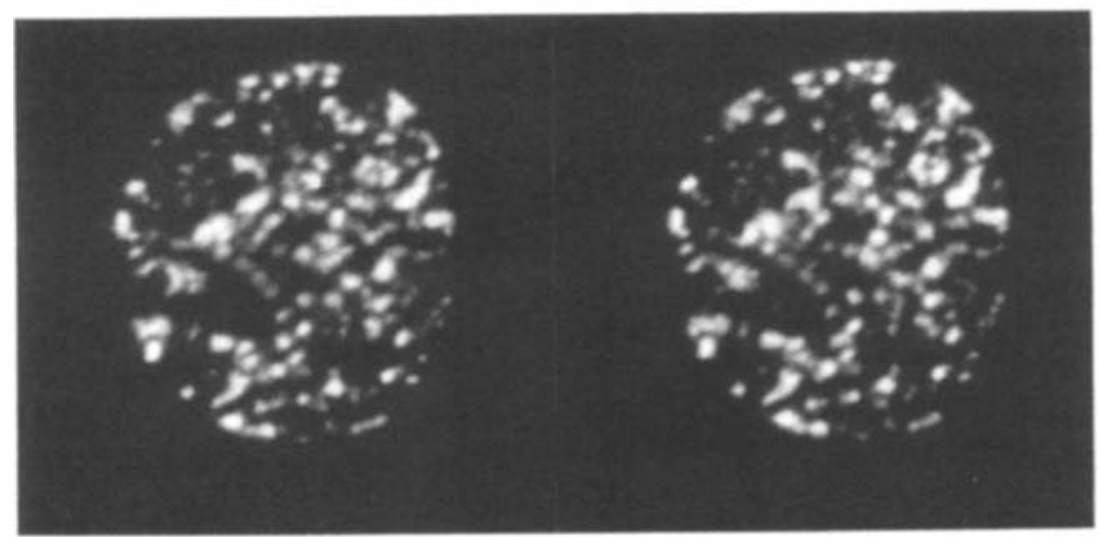

Figure 6 Three-dimensional reconstructed stereo image pair of a cell in very late S-phase replication. Cell labeling as in Figure 1 except with fluoresceinated antibody against BrdUrd and 4,6-diamidino-2-phenylindole (DAPI) for DNA. A Zeiss CLSM was used with excitation at $488 \mathrm{~nm}$ and emission $>515 \mathrm{~nm}$ and oil immersion planapo objective $63 \times / \mathrm{NA} 1.3$ with zoom factor 50. Six optical sections of the immunofluorescence at $0.4 \mu \mathrm{m}$ intervals were used for the reconstruction using the sterco visualization method described in Reference 168. 
through the cells. Most of the replication sites in the reconstructed stereo image can be seen to be localized on the nuclear envelope. Dual-wavelength CSLM images using a nuclear membrane marker (lamin) in conjunction with the BrdUrd antibody are being used to establish how closely the replication sites are associated with the nuclear membrane throughout S-phase (10).

Despite the positive record of performance, the existing confocal microscopes have problems that require attention. (a) The techniques for image acquisition are often optically and electronically inefficient. For example, the intensities of laser excitation required in the conf ocal mode often result in severe bleaching of the sample as optical sections are recorded, thereby biasing the data set or precluding a second pass for the recording of another fluorophore. In other instances, signal levels are high enough that the limited dynamic range of most frame-grabber circuits limits the accuracy of the subsequent quantitative analysis. (b) The $x, y$ registration of optical sections acquired sequentially with different excitation and emission filter combinations may be imprecise; thus the generation of ratio images may require registration corrections. (c) Combinations of probes with similar excitation spectra but distinct emission characteristics have been developed for use in flow cytometry and microscopy $(70,186,211)$. However, few microscope systems incorporate a provision for simultaneous multiparameter data acquisition. The following changes to CLSM systems could be effected with modest additional cost and would help to overcome some of the above deficiencies: use of better photomultipliers and photodiodes with higher quantum yield, variable bandwidth, and lower noise (e.g. through cooling); use of analog-to-digital converters with 10-14-bit resolution instead of the usual 8-bit resolution; simultaneous use of two or more detectors to acquire emission from distinct spectral regions isolated via dichroic filters or other dispersion systems, and storage of the data in separate frame buffers; and definition of restricted scan regions by signal threshold gating. Other potential advanced features are discussed below.

\section{DATA FLOW IN DIGITAL IMAGING MICROSCOPY}

\section{Data Acquisition}

The complexity of system design and implementation requires that we limit ourselves to some general observations. There are two approaches for recording image data: analog recording with a video recorder or optical disk, the method of choice for time-lapse experiments, used extensively with video cameras (95), and digital recording by frame-grabbing (available for video format or slow-scan camera signals as well as for photomultiplier sensors) or direct parallel transfer to magnetic disk. An inte- 
grated-systems philosophy is required, particularly with respect to control of the microscope, real-time data display and manipulation, data archiving, image processing, and communication with other remote computer services. A diagram of the configuration currently favored in our laboratory is shown in Figure 7. We stress the following points in particular.

DATA-ACQUISITION ELECTRONICS Most digital cameras are offered as integrated systems with 12-16-bit resolution and use standard interfaces to other computers. The laser scanning microscopes are similar in principle. Unfortunately, as we have already stated, most of the commercial instruments incorporate electronics originally intended for video signals of low dynamic range. Therefore, we are implementing in our CLSM a front-end system with the following characteristics: analog processing with alternative provisions for gated integration, determination of phase and amplitude (for modulated laser sources), adjustable electronic and multiple spectral bandwidths, and photon counting; analog-to-digital conversion with 12-bit resolution at $1-10 \mathrm{MHz}$ data-acquisition rates; some digital processing capabilities, including gating according to thresholded regions of interest; and real-time multiwindow display.

STORAGE OF IMAGE DATA Digital imaging necessarily involves vast storage requirements, particularly as formats, resolution, and the number of measurable parameters increase. Data compression algorithms can alleviate but not eliminate the problem. Random-access media are absolutely

Standard Port (IEEE)

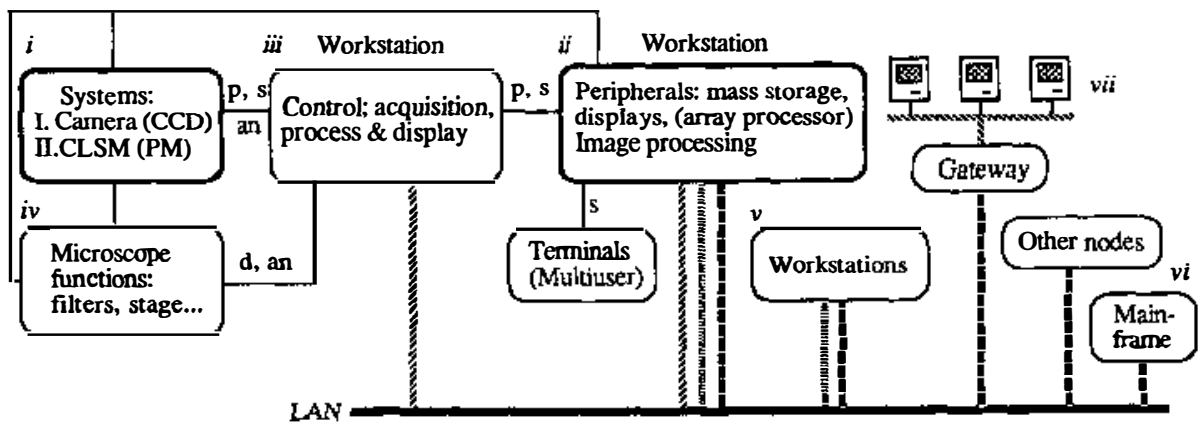

Figure 7 LDIM system. The CCD camera and CSLM are represented as complete subsystems $(i)$ which, together with a workstation (ii), constitute a minimal LDIM configuration. The control of image acquisition and auxiliary microscope functions can be either local (iii) or remote (iv). Communication over the local area network (LAN) is via multiprocessor cluster or other protocols (shown as different line patterns) to subsidiary workstations $(v)$, remote nodes and mainf rame computer (vi), and other processor networks (vii). Connections: $p$, parallel; $s$, serial; an, analog, $d$, digital. 
essential if processing is to be at all efficient. We favor the combination of a moderately large magnetic disk unit (capacity of $\sim 1$ gigabyte) and complementary optical recording drives. The current 5.25-inch disks are inexpensive, have capacities of 0.4-1 gigabyte, and are available in noneraseable versions useful for archiving, or, most recently, as eraseable storage media with very fast transfer rates.

\section{Data Display}

Display system requirements differ with respect to format and speed at the time of data acquisition and during the subsequent image processing. In direct association with the camera or microscope, digital imaging systems usually require the capability of displaying line scans, subimages, and superposed luminescence and other signals. The computer-graphics display of three-dimensional biological structures is a general problem (25, $40,72,104,120,138,179,198,222,232)$. In confocal microscopy, methods for extended-focus, auto-focus, and stereographic representations have received particular attention (Table 1; 28, 116, 168, 193, 209, 231). Stereo pairs can be viewed by anaglyphic (red-green) or polarized (liquid-crystal or dual-projector) image presentation techniques, by rapid generation of rotation sequences (a highly effective but technically demanding method) (72), and by generation of an artificial shadow or luminescence image (209). Other microscope systems which stress temporal resolution and differential viewing require rapid image processing (subtraction or ratio calculation). The display systems and monitors should offer pseudocolor coding of single or multiple overlayed images, sufficient random-access memory (RAM) for rapid sequence presentation, pan and zooming functions, and adequate pixel resolution $(512 \times 512$ or, preferably, $1024 \times$ 1024). Photographic, analog, or digital printers can be used to generate hard copy.

\section{Image Processing and Analysis}

Image processing considerations apply at three levels in the system. If the data are acquired with integrated camera or microscope systems, the firmware and command structures resident in a host computer dictate the nature of the operations. Following intermediate or ultimate storage of the image data in a standard format with well-defined headers and data structure, one can resort to a myriad of packaged sof tware systems for image processing, enhancement, analysis, and display. Important considerations are the programming language, flexibility in terms of integrating user-specific routines, speed (also a function of the hardware available, e.g. the array processors), and a convenient command structure with intelligent macro capabilities. We have used extensively the TCL-IMAGE 
processing software developed at the Delft Center for Image Processing of the Technical University of Delft, the Netherlands and currently available through Multihouse TSI, Amsterdam in versions for many mainframes, minicomputers, and workstations.

The image processing strategy is dictated by the particular application. As an example, we indicate in Figure 8 the scheme adopted for the pbFRET-DIM measurements presented earlier in Figure 2.

Not least of the problems associated with optical sectioning techniques in digital microscopy is that of three-dimensional restoration, basically the analysis of the corresponding three-dimensional OTF. Great progress has been made in the development of algorithms for restoring useful images of extended structures in medical imaging $(72,89)$ and microscopy $(1,2$, $36,111,120,127,136,141,214,232)$. Particularly intriguing are the restoration techniques based on maximum likelihood methods or the principle of maximum entropy (PME), in which the physical properties of the imaging device and associated noise components are used explicitly $(64$, 145). Additional effort is required to effect accurate quantitative analyses, as opposed to enhancement and restoration per se, of arbitrary grey-scale voxel distributions (94).

\section{LUMINESCENCE PROBES AND APPLICATIONS}

The measurement of luminescence phenomena with digital microscopes requires probes specific for the system or molecules of interest. Fortunately, the array of available fluorophores is growing rapidly. The wide

\begin{tabular}{|c|c|}
\hline $\begin{array}{ll}I & \text { record images } \\
\text {-I(d,0): } & \text { donor, initial } \\
\text {-I(a,0): } & \text { acceptor, initial } \\
\text {-I(d,t): } & \text { image during bleach } \\
\text {-I(d,int): } & \text { donor, bleaching } \\
\text {-I(a,f): } & \text { acceptor, final }\end{array}$ & $\begin{array}{l}\text { corrections } \\
\text { •bias/dark current } \\
\text {-flatness } \\
\text { •background } \\
\text {-registration }\end{array}$ \\
\hline $\begin{array}{l}\text { IV energy transfer } \\
\text {-energy transfer } \\
E=1-\phi \% \phi_{0} \\
\text { •energy transfer } \\
E=1-\tau_{\mathrm{bl}} / \tau_{\mathrm{bl}}\end{array}$ & $\begin{array}{l}\text { functions } \\
\text { •calculate ratio } \phi \\
\phi^{\prime}=\mathrm{I}(\mathrm{d}, 0) / \mathrm{I}(\mathrm{d}, \mathrm{int}) \\
\text { •calculate } \tau_{\mathrm{bl}} \\
\ln [\mathrm{I}(\mathrm{d}, \mathrm{t})] \text { vs time }\end{array}$ \\
\hline
\end{tabular}

Figure 8 Image processing scheme for pbFRET-DIM. The $I(a, 0)$ and $I(a, f)$ images are optional controls and are not required for the calculation of the transfer efficiency $E$. Operations are in the order $I, I I, I I I, I V$. 
range of spectral excitation and emission wavelengths facilitates the simultaneous use of multiple probes. Table 2 classifies some types of probes used in LDIM and their corresponding targets. An extensive handbook of commercially available luminescence reagents is available from Molecular Probes (Eugene, Oregon) (85). Other reviews are scattered in the literature (e.g. 195, 215). An important aspect of probe selection and sensitivity is related to labeling strategies. Small molecules may be introduced into living cells by passive or active transport through the cell membrane, by osmotic shock uptake, or by delivery through liposome fusion. Macromolecules may be introduced by capillary microinjection, by electroporation, or by endocytosis or phagocytosis induced, for example, by calcium phosphate or by scrape loading techniques (50). In addition, the plasma membrane of fixed cells is generally permeable and available to antibodies or small molecules. Accessibility may depend upon factors such as condensation, protein denaturation, and cross-linking of cellular components by the fixation procedure itself.

The power of LDIM lies in the correlated quantitative and morphometric information that can be derived about a variety of cellular functions. In one study (50), five luminescence parameters were measured simultaneously during wound healing in living cells. In another study, highresolution three-dimensional mapping using CLSM with three fluorescence probes and phase contrast was used to define the interaction of macromolecular assemblies in the protozoan Euplotes (158; D. Olins, A. Olins, M. Robert-Nicoud, T. M. Jovin, J. Wehland \& K. Weber, unpublished data). The three-dimensional organization of the interphase nucleus is the object of present intensive study using multiple markers; specific areas under investigation include DNA distribution $(30,93)$, replication loci in relation to nuclear antigens and DNA distribution $(8,10$; Figure 6), centromere and snRNP localizations (212), and chromosome-specific loci studied through fluorescence in situ hybridization $(132,147,207)$.

\section{Exploitation of the Photophysics of Luminescence Probes}

Although most microscopy based on emission is carried out with steadystate illumination and broadband detection of spectral emission, numerous photophysical manifestations of luminescence can be exploited in the microscope with present-day technology. The following discussion augments references to these matters already made above.

QUANTUM YIELD A probe may show a relative change in fluorescence intensity in response to a property of the local environment (e.g. polarity, viscosity, electric field) or upon binding to a particular macromolecule [e.g. DNA (8)] or to ions such as $\mathrm{H}^{+}, \mathrm{K}^{+}$, and $\mathrm{Ca}^{2+}$ (Table 2; 34, 203). 
Table 2 Cellular localization, properties, and applications of probes for LDIM ${ }^{\mathrm{a}}$

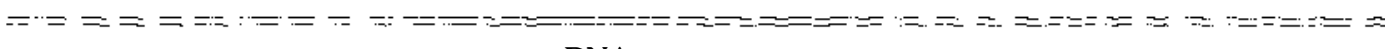

\section{DNA,}

Class of probes ${ }^{\mathrm{b}}$

Organelles

RNA

Membrane

$\mathrm{pH} \quad$ Cytoskeleton

Ions

Cytoplasm

References

Intrinsic luminescence (pigments, cofactors)

Small luminescent molecules

Fluorogenic substrates

Indirect probes (antibodies, ligands, hybridization probes)

$+$

$+$

$+$

$+$

Labeled cellular constituents

Photoactivated probes (photochromic and caged compounds)

Environment-sensitive probes Temporally resolved probes (triplet probes, lanthanides) Immunocolloidal gold or silver Bio- or chemiluminescence

+
+
+
+

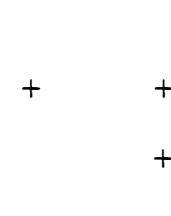

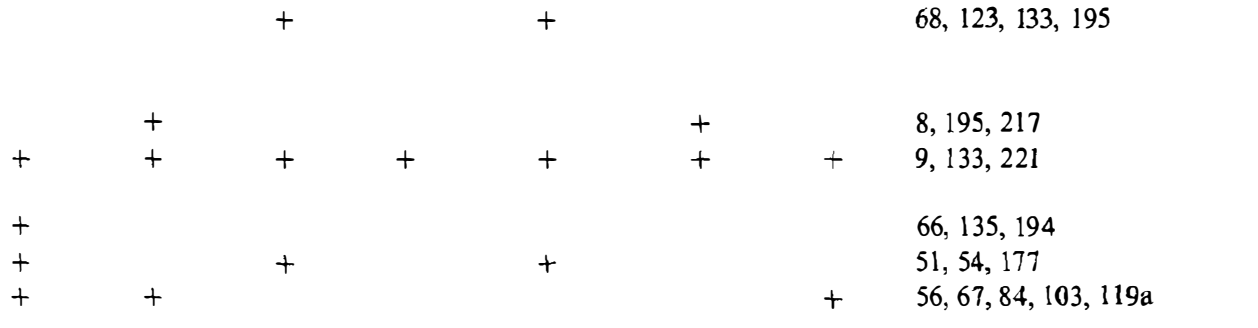

$112,164,213$

$30,34,41,55,93,124,128$,

$143,195,203,228,239$

$+\quad 129,204$

$31,32,80,105,147,148,162$, $163,212,218-220$

$68,123,133,195$
${ }^{\mathrm{a}}$ Several entries include references to studies of nonluminescence DIM. LDIM has been applied to the quantitation of macromolecules in electrophoretic gels using some of these probes.

${ }^{\circ}$ Reference 85 lists an extensive bibliography for LDIM applications of probes in all of these categories. 
Changes in membrane potential induced by external electric fields have been measured by video FDIM using a charge-shift potentiometric dye (78) and pulsed-laser excitation (239).

SPECTRAL DOMAIN The excitation and emission spectra of fluorophores are of ten strongly influenced by the environment of the probe [as shown for example by Prodan-actin undergoing polymerization from the $G$ to the $\mathrm{F}$ form (133)], the binding of ligands (Table 2), and cellular electrical activity (76). Such probes are best exploited in the microscope by acquisition of dual excitation and emission wavelengths either through motordriven selective filters and ratio imaging [pixel-by-pixel division of two digitized images $(34,123,124)$ ] or by diode array spectrographs $(156,164)$ or spectrum analyzers; the latter acquisition procedure is necessarily slower than the former.

TEMPORAL DOMAIN Fluorescence recovery after photobleaching (FRAP) was developed as a time-resolved fluorescence microscopic measurement in the 1970 s and has been extended to FDIM more recently $(20,97,181)$. For example, combined MCP intensifier-video detector systems have been used to study the translational movement of cell surface and cytoplasmic components. The lifetime of a fluorophore is a fundamental property and is accessible in the microscope with single-cell samples using pulsed light sources $(156,213)$ or phase-sensitive detection as we have shown here in the measurements of delayed luminescence. As already discussed, the microenvironment and competition by physical processes such as energy transfer will in general alter the fluorescence lifetime and photobleaching kinetics of a fluorophore.

FREQUENCY DOMAIN Emission signals excited by modulated light sources have a phase shift and a modulation depth characteristic for the probe(s) (74). By selecting an appropriate relative phase or harmonic component, one can achieve selectivity for a specific component with a particular lif etime and/or reject background contributions such as scattering and autofluorescence $(52,194,202)$. This method will undoubtedly find an important niche in LDIM.

SPATIAL DOMAIN The system devised for video-FRAP (97) can also be used for nonphotobleaching time-resolved spatial photometry (see 239). FRET provides information about molecular interactions over distances two orders of magnitude below the optical resolution of the microscope.

Determinations of movement and position with picometer to nanometer resolution have been achieved using colloidal gold particles and videoenhanced darkfield (51) or epipolarization (61) microscopy; protein-coated plastic microspheres and video-enhanced DIC microscopy (68); hair 
bundles on auditory cells and interferometrical fluctuation analysis (53); computer-assisted optical metrology (171); individual liganded low-density lipoprotein (LDL) receptors and video FDIM (79) [this and other (78) receptor systems can be examined after electrophoretic redistribution]; light scattering with video-enhanced microscopy [a theoretical study (144)]; and total internal reflection microscopy (TIRF), mentioned above. In addition, holographic imaging in a transient detection microscope has achieved micron resolution (48). While only some of these and other approaches cited earlier in this review are based on luminescence phenomena, they are best regarded as complementary to LDIM or as potential areas for its expansion.

POLARIZATION The quantitative determination of emission polarization involves combinations of signals and therefore constitutes another ratio imaging technique. The potentials in LDIM (221) have yet to be realized.

\section{QUO VADIS?}

We have emphasized the existing versatility and potential for further development of LDIM instrumentation and applications. At least three refinements of the microscopes would be of great utility: (a) development of a fast, electrooptical confocal LDIM with random-access capabilities within the object field; $(b)$ development of an optical accessory with which conventional fluorescence microscopes could be rendered confocal; and (c) integration of a high-speed automatic cell or particle sorting capability with LDIM [a low-speed LDIM sorter is presently available (167)]. In addition, new optical imaging principles need to be examined, such as those that operate in the transform domain (180) or are coupled to photothermal (33), magnetic resonance (117), or nonlinear optical (119) phenomena. Light could also be better exploited as a more active component of the overall system, as exemplified by the photoactivation of caged or other photochemical probes and by the astonishingly effective optical trapping and manipulation of cells and subcellular organelles by infrared laser beams (12).

The goal in LDIM with respect to sensitivity is single molecule detection. Its achievement will depend more upon the reduction of background contributions that limit selectivity and contrast than upon absolute light level. The instruments based on solid-state cameras and on beam scanning techniques both offer the required technology.

We have considered LDIM primarily in its relation to the concerns of basic scicnce. However, LDIM has much to offer in clinical medicine. This potential has been well documented in applications exploiting the optical 
sectioning capabilities of the confocal microscope $(15,182)$, multicolor imaging based on diode array detectors (191), the penetration of bone and imaging by infrared radiation (77), and fiber optic coupling in numerous intravital situations.

\section{ACKNOWLEDGMENTS}

We thank R. M. Clegg, G. Marriott, M. Robert-Nicoud, and T. Schormann for collaborations and helpful discussions, the German Research Council (DFG) for a grant for the confocal laser scanning microscope, Photometrics Ltd for providing the CCD camera system, and numerous colleagues for providing unpublished material and for critical reading of this manuscript.

\section{Literature Cited}

1. Agard, D. A. 1984. Annu. Rev. Biophys. Bioeng. 13: 191-219

2. Agard, D. A., Hiraoka, Y., Shaw, P., Sedat, J. W. 1988. See Ref. 216, pp. 353-77

3. Aikens, R. S., Agard, D. A., Sedat, J. W. 1988. See Ref. 216, pp. 292-314

4. Allen, R. D. 1985. Annu. Rev. Biophys. Biophys. Chem. 14: 265-90

5. Altkorn, R., Zare, R. N. 1984. Annu. Rev. Phys. Chem. 35: 265-89

6. Deleted in proof

7. Arndt-Jovin, D. J., Jovin, T. M. 1977. J. Histochem. Cytochem. 25: 585-89

8. Arndt-Jovin, D. J., Jovin, T. M. 1988. See Ref. 196, pp. 417-48

9. Arndt-Jovin, D. J., Robert-Nicoud, M., Kaufman, S. J., Jovin, T. M. 1985. Science 230: 247-56

10. Arndt-Jovin, D. J., Robert-Nicoud, M., Schormann, T., Jovin, T. M. 1988. Cytometry Suppl. 2: 59

11. Ash, E. A. 1980. Scanned Image Microscopy. New York: Academic

12. Ashkin, A., Dziedzic, J. M., Yamane, T. 1987. Nature 330: 769-71

13. Aslund, N., Lil jeborg, A., Forsgren, P.-O., Wahlsten, S. 1987. Scanning 9: 227-35

14. Axelrod, D., Burghardt, T. P., Thompson, N. L. 1984. Annu. Rev. Biophys. Bioeng. 13: 247-68

15. Bailey, G. W., ed. 1988. Proc. 46th Annu. Meet. Electron Microsc. Soc. Am., Milwaukee, Wis. San Francisco: San Francisco Press

16. Barry, B., Mathies, R. A., Pardoen, J. A., Lugtenburg, J. 1987. Biophys. J. 52: $603-10$
17. Bartels, P. H., Layton, J., Shoemaker, R. L. 1984. Monogr. Clin. Cytol. 9: 2861

18. Bayley, P. M., Dale, R. E., eds. 1985. Spectroscopy and the Dynamics of Molecular Biological Systems. Orlando, Fla: Academic

19. Benedetti, P. A., Evangelista, V., Giordano, P. A., Guidarini, D. 1988. Cytometry Suppl. 2: 193

20. Benson, D. M., Bryan, J., Plant, A. L. Gotto, A. M., Smith, L. C. 1985. J. Cell Biol. 100: 1309-23

21. Benson, D. M., Knopp, J. A. 1984. Photochem. Photobiol. 39: 495-502

22. Berns, M. W. 1979. Cell Biophys. I: 113

23. Bilhorn, R. B., Epperson, P. M., Sweedler, J. V., Denton, M. B. 1987. Appl. Spectrosc. 41: 1125-36

24. Bilhorn, R. B., Sweedler, J. V., Epperson, P. M., Denton, M. B. 1987. Appl. Spectropsc. 41: 1114-25

25. Böcker, F. R. P., Witte, G., Höhne, K. H. 1986. In Pictorial Information Systems in Medicine, NATO ASI Ser., ed. K. H. Höhne, pp. 469-84. Heidelberg: Springer

26. Born, M., Wolf, E. 1980. Principles of Optics. Elmsford, NY: Pergamon. 6th ed.

27. Boyde, A. 1986. Ann. NY Acad. Sci. 483: 428-39

28. Boyde, A. 1987. J. Microsc. 146: 13742

29. Brakenhoff, G. J., Blom, P., Barends, P. 1979. J. Microsc. 117: 219-32

30. Brakenhoff, G. J., van der Voort, H. T. M., van Spronsen, E. A., Linnemans, 
W. A. M., Nanninga, N. 1985. Nature 317: 748-49

31. Brakenhoff, G. J., van der Voort, H. T. M., van Spronsen, E. A., Nanninga, N. 1986. Ann. NY Acad. Sci. 483: 40515

32. Brakenhoff, G. J., van der Voort, H. T. M., van Spronsen, E. A., Nanninga, N. 1988. Scanning Microsc. 2: 33-40

33. Braslavsky, S. E., Heihoff, K. 1989. In Handbook of Organic Photochemistry, ed. J. C. Scaiano. Cleveland, Ohio: CRC. In press

34. Bright, G. R., Fisher, G. W., Rogowska, J., Taylor, D. L. 1987. J. Cell Biol. 104: 1019-33

35. Bustamante, C., Maestre, M. F. 1988. Proc. Natl. Acad. Sci. USA 85: 848286

36. Carrington, W., Fogarty, K. 1982. In IEEE Proc. Annu. Northeast Bioeng. Conf. 13: 108-11

37. Castleman, K. R. 1979. Digital Image Processing. Englewood Cliffs, NJ: Prentice-Hall

38. Chan, S. S., Arndt-Jovin, D. J., Jovin, T. M. 1979. J. Histochem. Cytochem. 27: $56-64$

39. Chellappa, R., Sawchuk, A. A., eds. 1985. Digital Image Processing and Analysis, Vols. 1, 2. Silver Spring, Md: IEEE Comput. Soc.

40. Coggins, J., Fogarty, K., Fay, F. 1986. Comput. Methods Programs Biomed. 22: $69 \cdots 77$

41. Connor, J. A. 1986. Proc. Natl. Acad. Sci. USA 83: 6178-83

42. Corin, A. F., Jovin, T. M. 1986. Biochemistry 25: 3995-4007

43. Corle, T. R., Chou, C.-H., Kino, G. S. 1986. Opt. Lett. 11:770-72

44. Cox, I. J. 1984. J. Microsc. 133: 14954

45. Cox, I. J., Sheppard, C. J. R. 1986. J. Opt. Soc. Am. A 3: 1152-58

46. Cox, I. J., Sheppard, C. J. R., Wilson, T. 1982. Optik Stuttgart 60: 391-96

47. Cram, L. S., Bartholdi, M. F., Wheeless, L. L. Jr., Gray, J. W. 1985. See Ref. 211, pp. 164-94

48. Cudney, R. S., Pierce, R. M., Feinberg, J. 1988. Nature 332: 424-26

49. Dale, R. E. 1988. In Polarized Spectroscopy of Ordered Systems, NATO ASI Ser. $C$, ed. B. Samori, E. W. Thrulstrup, pp. 491-567. Dordrecht, the Netherlands: Reidel

50. DeBiasio, R., Bright, G. R., Ernst, L. A., Waggoner, A. S., Taylor, D. L. 1987. J. Cell Biol. 105: 1613-22

51. DeBrabander, M., Geuens, G., Nuydens, R., Moeremans, M., DeMey, J. 1985. Cytobios 43: 273-83
52. Demas, J. N., Keller, R. A. 1985. Anal. Chem. 57: 538-45

53. Denk, W., Webb, W. W., Hudspeth, A. J. 1986. Biophys. J. 49: 21a

54. DeWaele, M., Renmans, W., Segers, E., Jochmans, K., Van Camp, B. 1988. J. Histochem. Cytochem. 36: 679-83

55. DeWeer, P., Salzberg, B. M., eds. 1986. Optical Methods in Cell Physiology. New York: Wiley-Intersci.

56. De Wet, J. R., Wood, K. V., de Luca, M., Helinski, D. R., Suhramani, S. 1987. Mol. Cell. Biol. 7: 725

57. Dougherty, E. E., Giardina, C. R. 1987. Image Processing-Continuous to Discrete: Geometric, Transform, and Statistical Methods. Englewood Clifts, NJ: Prentice-Hall

58. Draaijer, A., Houpt, P. M. 1988. Scanning 10: 139-45

59. Duncan, M. D., Reint jes, J., Manuccia, T. J. 1985. Opt. Eng. 24: 352-55

60. Egger, M. D., Petran, M. 1967. Science 157: $305-7$

61. Ellis, I. O., Bell, J., Bancroft, J. D. 1988. J. Histochem. Cytochem. 36: 121-24

62. Elson, E. L. 1985. Annu. Rev. Phys. Chem. 36: 379-406

63. English, R. E. Jr., George, N. 1987. Appl. Opt. 26: 2360-63

64. Frieden, B. R. 1987. Appl. Opt. 26: 1755-64

65. Galbraith, W., Ryan, K. W., Gliksman, N., Taylor, D. L., Waggoner, A. S. 1989. Comput. Med. Imaging Graph. 13: 47-60

66. Garland, P. B., Johnson, P. 1985. See Ref. 18, pp. 95-118

67. Geiger, R., Misha, W. 1987. J. Clin. Chem. Clin. Biochem. 25: 31-38

68. Gelles, J., Schnapp, B. J., Sheetz, M. P. 1988. Nature 331: 450-53

69. Gingell, D., Heavens, O. S., Mellor, J. S. 1987. J. Cell Sci. 87: 677-93

70. Glaser, A. N., Stryer, L. 1984. Trends Biochem. Sci. 1984: 423-25

71. Göhde, W., Dittrich, W. 1971. Acta Histochem. Suppl. 10: 42-51

72. Goodman, J. W. 1968. Introduction to Fourier Optics. San Francisco: McGraw-Hill

73. Goldwasser, S. M., Reynolds, R. A. 1987. Comput. Vis. Graph. Image Process. $39: 1-27$

74. Gratton, E., Jameson, D. M., Hall, R. D. 1984. Annu. Rev. Biophys. Bioeng. 13: $105-24$

75. Griffith, O. H., Rempfer, G. F. 1985. Annu. Rev. Biophys. Biophys. Chem. 14: $113-30$

76. Grinvald, A. 1985. Annu. Rev. Neurosci. 8: 263-305

77. Grinvald, A., Lieke, E., Frostig, R. D., 
Gilbert, C. D., Wiesel, T. N. 1986. Nature 324: 36i-64

78. Gross, D., Loew, L. M., Webb, W. W. 1986. Biophys. J. 50: 339-48

79. Gross, D., Webb, W. W. 1986. Biophys. J. 49: $901-11$

80. Gross, D., Webb, W. W. 1988. In Spectrosco pic Membrane Probes, ed. L. M. Loew. Boca Raton, Fla: CRC

81. Delcted in proof

82. Hansen, E. W., Allen, R. D., Strohbehn, J. W., Chaffee, M. A., Farrington, D. L., et al. 1985. J. Microsc. 140: 371-81

83. Harvey, E. N., Chase, A. M. 1942. Rev. Sci. Instrum. 13: 36568

84. Hauber, R., Geiger, R. 1988. Nucleic Acids Res. 16: 1213

85. Haugland, R. P. 1988. Handbook of Fluorescent Probes and Research Chemicals, No. 2. Eugene, Ore: Mol. Probes

86. Hayakawa, T., Kinoshita, K., Miyaki, S., Fujiwake, H., Ohsuka, S. 1986. Photochem. Photobiol. 43: 95-97

87. Herman, B. 1988. See Ref. 196, pp. 220-45

88. Herman, B., Fernandez, S. 1982. Biochemistry 21: 3275-83

89. Herman, G. T. 1986. Annu. Rev. Comput. Sci. 1: 153-79

90. Hiraoka, Y., Sedat, J. W., Agard, D. A. 1987. Science 238: 36-41

91. Hirschfeld, T. 1977. Appl. Spectrosc. 31: 245

92. Hirschfeld, T., Block, M. J., Mueller, W. 1977. J. Histochem. Cytochem. 25: 719

93. Hochstrasser, M., Sedat, J. W. 1987. J. Cell Biol. 104: 1455-83

94. Höhne, K. H., Bernstein, R. 1986. IEEE Trans. Med. Imaging 5: 45-47

94a. Howard, J. N., ed. 1987. Appl. Opt. 26(16): 3199-3416

95. Inoué, S. 1986. Video Microscopy. New York: Plenum

96. Isaacson, M., Betzig, E., Harootunian, A., Lewis, A. 1986. Ann. NY Acad. Sci. 483: 448-56

97. Jacobson, K., Ishihara, A., Inman, R. 1987. Annu. Rev. Physiol. 49: 16375

98. Janesick, J. R., ed. 1987. Opt. Eng. 26(8-10): 685-1083

99. Janssen, G. C. A. M., Rousseeuw, B. A. C., van der Voort, H. T. M. 1987. Rev. Sci. Instrum. 58: 598-99

100. Jarvis, L. R. 1988. J. Microsc. 150: 8397

101. Jericevic, Z., Benson, D. M., Bryan, J., Smith, L. C. 1988. J. Microsc. 149:23345

102. Jericevic, Z., Wiese, B., Bryan, J.,
Smith, L. C. 1988. See Ref. 196, pp. 4783

103. Johnson, C. H., Inoué, S., Flint, A., Hastings, J. W. 1985. J. Cell Biol. 100: $1435-46$

104. Joshi, H. C., Chu, D., Buxbaum, R. E., Heidemann, S. R. 1985. J. Cell Biol. 101: 697-705

105. Jovin, T. M., Arndt-Jovin, D. J. 1989. See Ref. 112. In press

105a. Jovin, T. M., Marriott, G., Clegg, R. M., Arndt-Jovin, D. J. 1989. Ber. Bunsenges. Phys. Chem. In press

106. Jovin, T. M., Robert-Nicoud, M., Arndt-Jovin, D. J., Schormann, T. 1988. See Ref. 15, pp. 96-97

107. Jovin, T. M., Vaz, W. L. C. 1989. Methods Enzymol. 172: 471-513

108. Jungerman, R. L., Hobbs, P. C. D., Kino, G. S. 1984. Appl. Phys. Lett. 45 846-48

109. Kam, Z. 1987. Q. Rev. Biophys. 20: 201-59

110. Kaufmann, G. I., Nester, J. F., Wasserman, D. E. 1971. J. Histochem. Cytochem. 19: 469-76

111. Kawata, S., Nakamura, O., Minami, S. 1987. J. Opt. Soc. Am. A 4: 292-97

112. Kohen, E., Ploem, J. S., Hirschberg, J. G., eds. 1989. Cell Structure and Function by Microspectrofluorometry. Orlando, Fla: Academic. In press

113. Koppel, D. E. 1979. Biophys. J. 28: 281-92

114. Koppel, D. E., Axelrod, D., Schlessinger, J., Elson, E. L., Webb, W. W. 1976. Biophys. J. 16: 1315-29

115. Kuttner, P. 1986. Opt. Eng. 25: 180-83

116. Kuwahara, M., Eiho, S., Baba, K. 1986. Photonics Spectra 1986(12): 93 96

117. Kwiram, A. L., Ross, J. B. A. 1982. Annu. Rev. Biophys. Bioeng. 11: 23349

118. Larrabee, R. D. 1988. See Ref. 15, pp. $50-51$

119. Laubereau, A. 1988. Top. Appl. Phys. 60: 38-112

119a. Leaback, D. H., Haggart, R. 1989. Proc. Sth Int. Symp. Biolumin. Chem. Fluoresc., Florence, Italy, 1988. New York: Wiley. In press

120. Lenz, R. 1986. PhD thesis. Univ. Linkoeping, Sweden

121. Leplock, J. R., Thompson, J. E., Kruuv, J., Wallach, D. F. H. 1978. Biochem. Biophys. Res. Commun. 85: 34450

122. Li, Y. 1987. J. Opt. Soc. Am. A 4: 1349 53

123. Luby-Phelps, K., Lanni, F., Taylor, D. L. 1988. Annu. Rev. Biophys. Biophys. Chem. 17: 369-96 
124. Luby-Phelps, K., Taylor, D. L., Lanni, F. 1986. J. Cell Biol. 102: 2015-22

125. Lukosz, W. 1966. J. Opt. Soc. Am. 56: 1463-72

126. Lukosz, W. 1967. J. Opt. Soc. Am. 57: $932-41$

127. Macias-Garza, F., Bovik, A. C., Diller, K. R. 1988. Opt. Eng. 27: 461-65

128. Mackay, C. D. 1986. In Electrophoresis '86, ed. M. J. Dunn, pp. 720-22. Weinheim, Fed. Rep. Germany: VCH

129. Mangel, W. F., Livingston, D. C., Brockelhurst, J. R., Cannon, J. F., Leytus, S. P., et al. 1981. Methods Enzymol. 80: 414-24

130. Mansuripur, M. 1986. J. Opt. Soc. Am. A 3: 2086-93

131. Manuccia, T. J., Loda, R. T. 1989. Ber. Bunsenges. Phys. Chem. In press

132. Manuelidis, L., Borden, J. 1988. Chromosoma 96: 397-410

133. Marriott, G., Zechel, K., Jovin, T. M. 1988. Biochemistry 27: 6214-20

134. Marshall, G. F., ed. 1985. Laser Beam Scanning: Opto-Mechanical Devices, Systems, and Data Storage Optics. New York: Dekker

135. Marshall, N. J., Dakubu, S., Jackson, T., Ekins, R. P. 1981. In Monoclonal Antibodies and Developments in Immunoassay, ed. A. Albertini, R. Ekins, pp. 101-8. Amsterdam: Elsevier

136. Martinez-Herrero, R., Sancha, S. G. 1988. Opt. Eng. 27: 456-60

137. Matey, J. R., Crandall, R. S., Brycki, B. 1987. Rev. Sci. Instrum. 58: 56770

138. Mathog, D., Hochstrasser, M., Sedat, J. W. 1985. J. Microsc. 137: 241-42

139. McCutchen, C. W. 1967. J. Opt. Soc. Am. 57: 1190-92

140. Melles Griot. 1988. Optics Guide 4. Irvine, Calif: Melles Griot

141. Merickel, M. 1988. Comput. Vis. Graph. Image Process. 42: 206-19

142. Mickols, W., Maestre, M. F. 1988. Rev. Sci. Instrum. 59: 867-72

143. Millard, P. J., Gross, D., Webb, W. W., Fewtrell, C. 1988. Proc. Natl. Acad. Sci. USA 85: 1854-58

144. Mizushima, Y. 1988. Appl. Opt. 27 2587-94

145. Mohammad-Djafari, A., Demoment, G. 1987. Appl. Opt. 26: 1745-54

146. Muchel, F. 1988. Zeiss Inf. 30: 20

147. Nederlof, P. M., Robinson, D., Wiegant, J., Hopman, A. H. N., Tanke, J. H., Raap, A. K. 1988. Cytometry Suppl. 2: 535

148. Deleted in proof

149. Petrán, M., Hadravsky, M. D., Egger, R., Galambos, J. 1968.J. Opt. Soc. Am. 58: $661-64$
150. Pieper, R. J., Park, J., Poon, T.-C. 1988. Appl. Opt. 27: 2040-47

151. Piller, H. 1977. Microscope Photometry. Berlin: Springer

152. Ploem, J. S. 1987. Appl. Opt. 26: 322631

153. Ploem, J.S. 1987. In Imaging and Visual Documentation in Medicine, ed. $\mathrm{K}$. Wamstcker, U. Jonas, G. Van der Veen, P. F. G. M. Van Waes, pp. 52336. Amsterdam: Elsevier

154. Preston, K. Jr. 1986. J. Histochem. Cytochem. 34: 67-74

155. Qian, H., Elson, E. L. 1988. See Ref. 15 , pp. 38-39

156. Ramponi, R., Rodgers, M. A. J. 1987. Photochem. Photobiol. 45: 161-65

157. Rigler, R., Rabl, C.-R., Jovin, T. M. 1974. Rev. Sci. Instrum. 45: 580-88

158. Robert-Nicoud, M., Arndt-Jovin, D. J., Schormann, T., Jovin, T. M. 1989. Eur. J. Cell Biol. In press

159. Rosenfeld, A. 1988. Comput. Vis. Graph. Image Process. 42: 234-93

160. Rosenfeld, A., Kak, A. C. 1982. Digital Image Processing. New York: $\Lambda$ cademic

161. Rudd, E. P., Mueller, R. K., Robbins, W. P., Skaar, T., Soumekh, B., Zhou, Z. Q. 1987. Rev. Sci. Instrum. 58: 4553

162. Salmon, E. D., Leslie, R. J., Saxton, W. M., Karow, M. L., McIntosh, J. R. 1984. J. Cell Biol. 99: 2165-74

163. Salmon, E. D., McKeel, M., Hays, T. 1984. J. Cell Biol. 99: 1066-75

164. Salmon, J. M., Kohen, E., Viallet, P., Hirschberg, J. G., Wouters, A. W., et al. 1982. Photochem. Photobiol. 36: 585-93

165. Sato, S., Ito, H., Inaba, H., Taguchi, Y., Kasai, M. 1986. Opt. Quantum Electron. 18: 81-84

166. Sawatari, T. 1973. Appl. Opt. 12: 2768 72

167. Schindler, M., Trosko, J. E., Wade, M. H. 1987. Methods Enzymol. 141: 439-49

168. Schormann, T., Robert-Nicoud, M., Jovin, T. M. 1989. Eur. J. Cell Biol. In press

169. See, C. W., Vaez-Iravani, M. 1988. Appl. Opt. 27: 2786-92

170. Seely, G. R. 1987. Biophys. J. 52: 31116

171. Seitz, P. 1988. Opt. Eng. 27: 535-40

172. Shack, R., Bartels, P. H., Buchroeder, D., Shoemaker, R. L., Hillman, D., Vukobratovich, D. 1987. Anal. Quant. Cytol. Histol. 9: 509-20

173. Sheetz, M. P., Koppel, D. E. 1979. Proc. Natl. Acad. Sci. USA 76: 331417 
174. Sheppard, C. J. R. 1988. J. Microsc. 149: 73-75

175. Sheppard, C. J. R., Choudhury, A. 1977. Opt. Acta 24: 1051-73

176. Sheppard, C. J. R., Matthews, H. J. 1987. J. Opt. Soc. Am. A 4: 1354-60

177. Shotton, D. M. 1988. J. Cell Sci. 89: 129-50

178. Shuman, H. 1988. J. Microsc. 149: 6771

179. Silage, D. A., Gil, J. 1985. J. Microsc. 138: 22 1-27

180. Simpson, M. J. 1987. Appl. Opt. 26: 1786-91

181. Smith, L. C., Benson, D. M., Gotto, A. M., Bryan, J. Jr. 1986. Methods Enzymol. 129: 857-73

182. Somlyo, A. P., ed. 1986. Recent Advances in Electron and Light Optical Imaging in Biology and Medicine. Ann. NY Acad. Sci. Vol. 483

183. Spring, K. R., Lowry, R. J. 1988. See Ref. 216, pp. 269-89

184. Spring, K. R., Smith, P. D. 1987. J Microsc. 47: 265-78

185. Steen, H. B., Lindmo, T. 1979. Science 204: 403-4

186. Steinkamp, J. A., Stewart, C. C., Crissman, H. A. 1982. Cytometry 2: 226-31

187. Stelzer, E. H. K. 1988. PhD thesis. Univ. Heidelberg, Fed. Rep. Germany

188. Stelzer, E. H. K., Stricker, R., Pick, R. Storz, C., Wijnaendts-van-Resandt, R. W. 1988. SPIE J. 909: 312-18

189. Stryer, L. 1978. Annu. Rev. Biochem. 47: 819-46

190. Suzuki, T., Horikawa, Y. 1986. Appl. Opt. 25: 4115-21

191. Svanberg, S. 1987. Phys. Scr. T19: 46975

192. Szabo, A. 1984. J. Chem. Phys. 81: $150-67$

193. Takamatsu, T., Fujita, S. 1988. J. Microsc. 149: 167-74

194. Tanke, H. J., van Schadewijk, A., van Gelderen, S., Beverloo, B. 1988. Cytometry Suppl. 2: 202

195. Taylor, D. L., Waggoner, A. S., Murphy, R. F., Lanni, F., Birge, R. R., eds. 1986. Applications of Fluorescence in the Biomedical Sciences. New York: Liss

196. Taylor, D. L., Wang, Y. L., eds. 1988. Fluorescence Microscopy of Living Cells in Culture. Fluorescent Analogs, Labeling Cells and Basic Microscope System. Methods Cell Biol. Vol. 30

197. Teague, E. C. 1988. See Ref. 15, pp. 1004-5

198. Tieman, D. G., Murphey, R. K., Schmidt, J. T., Tieman, S. B. 1986. J. Neurosci. Methods 17: 231-45

199. Tinoco, J. Jr., Mickols, W., Maestre,
M. F., Bustamante, C. 1987. Annu. Rev. Biophys. Biophys. Chem. 16: 319-49

200. Torroba, R., Garavaglia, M. 1987. Appl. Opt. 26: 2860-63

201. Tron, L., Szöllősi, J., Damjanovich, S., Helliwell, S. H., Arndt-Jovin, D. J., Jovin, T. M. 1984. Biophys. J. 45: 93946

202. Trukula, M., Keller, R. A. 1985. Anal. Chem. 57: 1663-69

203. Tsien, R. Y., Poenie, M. 1986. Trends Biochem. Sci. 1986: 450-55

204. Tsien, R. Y. 1988. See Ref. 196, pp. 127-56

205. Tsuchiya, Y., Inuzuka, E., Kurono, T., Hosoda, M. 1985. J. Imaging Technol. 11: $215-20$

206. Uster, P. S., Pagano, R. E. 1986. J. Cell Biol. 103: 1221-34

207. van Dekken, H., van Spronsen, E., Bauman, J., Jonker, R., Visser, J. 1988. Cytometry Suppl. 2: 58

208. van der Voort, H. T. M., Brakenhoff, G. J. 1988. Optik Stuttgart 78: 4853

209. van der Voort, H. T. M., Brakenhoff, G. J., Baarslag, M. W. 1989. J. Microsc. In press

210. van der Voort, H. T. M., Brakenhoff, G. J., Valkenburg, J. A. C., Nanninga, N. 1985. Scanning 7: 66-78

211. Van Dilla, M. A., Dean, P. N., Laerum, O. D., Melamed, M. R., eds. 1985. Flow Cytometry: Instrumentation and Data Analysis. New York: Academic

212. van Driel, R., de Jong, L., de Graaf, A., van der Voort, H. M., Brakenhoff, J. A., et al. 1988. Cytometry Suppl. 2: 192

213. Vigo, J., Salmon, J. M., Viallet, P. 1987. Rev. Sci. Instrum. 58: 1433-38

214. Vogel, R. H., Provencher, S. W. 1988. Ultramicroscopy 25: 223-40

215. Waggoner, A. S., DeBiasio, R., Conrad, P., Bright, G. R., Ernst, L., et al. 1988. See Ref. 196, pp. 449-78

216. Wang, Y. L., Taylor, D. L., eds. 1988. Quantitative Fluorescence Microscopy: Imaging and Spectroscopy. Methods Cell Biol. Vol. 29

217. Ware, B. R., Brvenik, L. J., Cummings, R. T., Furukawa, R. H., Krafft, G. A. 1986. See Ref. 195, pp. 141-58

218. Watts, T. H., Gaub, H. E., McConnell, H. M. 1986. Nature 320: 179-81

219. Watts, T. H., McConncll, H. M. 1986. Proc. Natl. Acad. Sci. USA 83: $9660-$ 64

220. Webb, W. W., Gross, D. 1986. See Ref. 195 , pp. $405-22$

221. Weber, G. 1986. See Ref. 195, pp. 60116

222. Weiss, I. 1988. Comput. Vis. Graph. Image Process. 41: 172-85 
223. White, J. G., Amos, W. B., Fordham, M. 1987. J. Cell Biol. 105: 41- 48

224. Wijnaendts-van-Resandt, R. W. 1987. SPIE J. 809: 101-6

225. Wijnaendts-van-Resandt, R. W., Marsman, H. J. B., Kaplan, R., Davoust, J., Stelzer, E. H. K., Stricker, R. 1985. J. Microsc. 138: 29-34

226. Wilke, V. 1985. Scanning 7: 88--96

227. Williams, C. S., Becklund, O. A. 1972. Optics: A Short Course for Engineers and Scientists. New York: Wiley-Intersci.

228. Williams, D. A., Fogarty, K. E., Tsien, R. Y., Fay, F. S. 1985. Nature 318: 55861

229. Wilson, T., Carlini, A. R. 1987. Opt. Lett. 12: 227-29

230. Wilson, T., Carlini, A. R. 1988. J. Microsc. 149: 51-66

231. Wilson, T., Sheppard, C. 1984. Theory and Practice of Scanning Optical Microscopy. New York: Academic

232. Wu, K., Hesselink, L. 1988. Appl. Opt. 27: $395-404$
233. Yoshida, T. M., Zarrin, F., Barisas, B. G. 1988. Biophys. J. 54: 277-88

234. Young, I. T. 1983. Applications of Digital Image Processing, ed. A. Oosterlinck, A. Tescher, 6: 326-35. Bellingham, Wash: Soc. Photo-Opt. Instrum. Eng.

235. Young, I. T. 1988. See Ref. 196, pp. 145

236. Young, I. T., Balasubramanian, Dunbar, D. L., Peverini, R. L., Bishop, R. P. 1982. IEEE Trans. Biomed. Eng. 29: $70-81$

237. Young, T. Y., Fu, K.-S., eds. 1986. Handbook of Pattern Recognition and Image Processing. Orlando, Fla: Academic

238. Yuan, P., Walt, D. R. 1987. Anal. Chem. 59: 2391-94

REFERENCE ADDED IN PROOF

239. Kinosita, K. Jr., Ashikawa, I., Hibino, M., Shigemori, M., Yoshimura, H., et al. 1988. SPIE J. 909: 271-77 


\section{CONTENTS}

\section{Structural Principles}

Thermodynamic Problems of Protein Structure, Peter L. Privalov 47

The $\beta$-Sheet to Coil Transition, Wayne L. Mattice 93

The Electrostatic Properties of Membranes, Stuart McLaughlin 113

Free Energy Via Molecular Simulation: Applications to

Chemical and Biomolecular Systems, D. L. Beveridge and

F. M. DiCapua

STRUCTURE AND FunCTION

Escherichia coli Aspartate Transcarbamoylase: Structure,

Energetics, and Catalytic and Regulatory Mechanisms, Norma M. Allewell

X-Ray Absorption Spectroscopic Investigations of Cytochrome $c$ Oxidase Structure and Function, Robert $A$. Scott

Biochemistry and Biophysics of Excitation-Contraction Coupling, Sidney Fleischer and Makoto Inui

Toward a Unified Model of Chromatin Folding, J. Widom

Structure and Function of the Red Blood Cell Anion

Transport Protein, Michael L. Jennings

\section{DYNAMICS}

Mechanism of Photosynthetic Water Oxidation, Gary W. Brudvig, Warren F. Beck, and Julio C. de Paula

The Study of Lipid Phase Transition Kinetics by Time-Resolved X-Ray Diffraction, $M$. Caffrey

Protein-Mediated Membrane Fusion, Toon Stegmann, Robert $W$. Doms, and Ari Helenius

EMERGING TECHNIQUES

Expanding Roles of Computers and Robotics in Biological Macromolecular Research, Akiyoshi Wada, Shun-ichi Kidokoro, and Shigeru Endo

Physical Studies of Protein-DNA Complexes by Footprinting, Thomas D. Tullius 
Properties and Uses of Photoreactive Caged Compounds, James A. McCray and David R. Trentham

Luminescence Digital Imaging Microscopy, Thomas M. Jovin and Donna J. Arndt-Jovin

Time-Resolved Macromolecular Crystallography, Keith Moffat

INDEXES

Subject Index

Cumulative Index of Contributing Authors, Volumes 14-18

Cumulative Index of Chapter Titles, Volumes 14-18 